\title{
Solving Black-Scholes equations using fractional generalized homotopy analysis method
}

\author{
S. R. Saratha ${ }^{1}$ - G. Sai Sundara Krishnan ${ }^{2} \cdot$ M. Bagyalakshmi ${ }^{3}$. Chee Peng Lim ${ }^{4}$
}

Received: 9 April 2020 / Revised: 2 August 2020 / Accepted: 14 August 2020 /

Published online: 4 September 2020

(c) SBMAC - Sociedade Brasileira de Matemática Aplicada e Computacional 2020

\begin{abstract}
This paper aims to solve the Black-Scholes (B-S) model for the European options pricing problem using a hybrid method called fractional generalized homotopy analysis method (FGHAM). The convergence region of the B-S model solutions are clearly identified using $h$-curve and the closed form series solutions are produced using FGHAM. To verify the convergence of the proposed series solutions, sequence of errors are obtained by estimating the deviation between the exact solution and the series solution, which is increased in number of terms in the series. The convergence of sequence of errors is verified using the convergence criteria and the results are graphically illustrated. Moreover, the FGHAM approach has overcome the difficulties of applying multiple integration and differentiation procedures while obtaining the solution using well-established methods such as homotopy analysis method and homotopy perturbation method. The computational efficiency of the proposed method is analyzed using a comparative study. The advantage of the proposed method is shown with a numerical example using the comparative study between FGHAM and Monte Carlo simulation. Using the numerical example, analytical expression for the implied volatility is derived and the non-local behavior is studied for the various values of the fractional parameter. The results of FGHAM are statistically validated with the exact solution and the other existing computational methods.
\end{abstract}

Keywords Fractional calculus · Laplace-type integral transform · Fractional partial differential equations · Mittag-Leffler function · Implied volatility · Monte Carlo simulation

Mathematics Subject Classification $65 \mathrm{H} 20 \cdot 35 \mathrm{G} 31 \cdot 35 \mathrm{C} 10 \cdot 26 \mathrm{~A} 33 \cdot 34 \mathrm{~A} 08 \cdot 35 \mathrm{R} 11$

\section{Introduction}

The Black-Scholes equations are the most popular mathematical models used for analyzing the pricing option problems in financial mathematics. Several authors have analyzed BS model using the various mathematical and numerical techniques, and proposed either closed form solution or numerical solution, respectively. Even though the BS model provides

Communicated by José Tenreiro Machado.

Extended author information available on the last page of the article 
reasonable solution to analyze pricing option problems, the governing BS equation was constructed under certain limitations of assuming various financial parameters such as volatility and risk-free rate as constants. Hence, determining the essential financial parameter 'implied volatility' for options cannot be estimated in closed form. To overcome the difficulty, many authors have proposed several types of BS models in different perspective. Rouah (2014) derived various types of Black-Scholes PDE for the option by considering hedging and Replication, etc. James and Garven (1986) has demonstrated an alternative derivation of the Black-Scholes option pricing formula based on the risk neutrality arguments.

Orlando and Taglialatela (2017) estimated the implied volatility for the options using both the closed form solutions, and numerical solutions and the results are computationally illustrated. This motivated to solve fractional BS equations through which the non-local behavior of option price and implied volatility can be analyzed. Fractional BS equations were initiated especially to study the fractal structure of the financial market. Wyss (2000) developed a fractional Black-Scholes equation with a time-fractional derivative to the price European call option. Jumarie (2008, 2010 derived the time- and space-fractional BlackScholes equations and studied the pricing problems for both the European and American put options and gave optimal fractional Merton's portfolio.

Recently, the theory and development of new computational methodology of solving fractional Black-Scholes partial differential equation has attracted the attention of many researchers over the past 2 decades. Company et al. (2008) applied a semi-discretization technique to solve the B-S option pricing consisting of partial differential equations. Gülkaç (2010) designed an homotopy perturbation method (HPM) to yield a solution for the B-S equation with the European option pricing problem. Grossinho and Morais (2013) introduced an upper and lower solutions method to solve the non-linear boundary value problem related to financial modeling. Lesmana and Wang (2013) developed an upwind finite-difference method to solve the non-linear B-S equation for governing the European option pricing problem. Song and Wang (2013) applied a finite-difference method to solve the fractional B-S option pricing model. Elbeleze et al. (2013) initiated a combination of HPM, Sumudu transform, and He's polynomials to produce a solution for the fractional B-S equation. Kumar et al. (2014) used both the HPM and homotopy analysis method (HAM) techniques to tackle the time-fractional B-S equation with boundary conditions pertaining to the European option pricing problem. Ghandehari and Ranjbar (2014) identified the Lagrange multipliers by means of the Sumudu transform to produce an accurate solution for the fractional B-S equation along with the initial condition for the European option pricing problem. Xiaozhong et al. (2016) proposed a universal difference method for handling the time-space-fractional B-S equation. Ravi Kanth and Aruna (2016) employed the fractional differential transform method and modified the fractional differential transform method to solve the time-fractional B-S European option pricing equation.

Khan and Ansari (2016) solved the fractional B-S European option pricing equation using the Sumudu transform and its derivatives. Granada et al. (2017) applied the HPM model to solve the B-S equation. Ouafoudi and Gao (2018) used both HPM and modified HPM and Sumudu transform to yield solutions for the B-S equation in the form of convergence power series with a regularly calculated element. Farhadi and Erjaee (2018) introduced a timefractional derivative for solving the B-S equation. Sawangtong et al. (2018) investigated an analytical solution for undertaking the B-S equation with two assets using the Laplace transform HPM approach in the Liouville-Caputo fractional derivative sense. Yavuz and Ozdemir (2018) initiated a conformable fractional adomian decomposition method (CFADM) and conformable fractional modified HPM to tackle the fractional B-S equation. Kittipoom (2018) applied an invariant subspace method to solve the time- and pricing-fractional B-S 
equation. Jena and Chakraverty (2019) introduced a residual power series (RPS) method for finding the analytical solution for the fractional B-S equation with an initial condition in the European option pricing problem. Prathumwan and Trachoo (2019) applied the Laplace HPM to yield an approximate solution for the B-S partial differential equation in the European put option with two assets. Uddin and Taufiq (2019) solved the time-fractional B-S equation through a transformation method with the radial basis kernel. Sumiati (2019) introduced the Laplace-Adomian decomposition method to solve the B-S equation.

Osman et al. (2018) investigated the coupled Schrodinger-Boussinesq equation with variable coefficients and used the unified method to obtain two categories of new non-autonomous complex wave solutions. Osman and Machado (2018) analyzed the $(2+1)$-dimensional Bogoyavlensky-Konopelchenko equation with variable coefficients via the generalized unified method. They also studied the propagation and dynamical behaviors of these solutions for the different choices of arbitrary variable coefficients. Rezazadeh et al. (2019) examined the hyperbolic rational solutions for four conformable fractional Boussinesq-like equations using the exponential rational function method. Osman et al. (2019a) applied the unified method to examine the $(3+1)$-dimensional conformable fractional Zakharov-Kuznetsov equation with power-law non-linearity and determined the following types of wave solutions: solitary, soliton, elliptic, and periodic (hyperbolic) wave rational solutions. Ghanbari et al. (2019) proposed new analytical obliquely propagating wave solutions for the time-fractional extended Zakharov-Kuzetsov equation with conformable derivative. Osman (2019) analytically studied the water wave solutions for the coupled fractional variant Boussinesq equation and investigated the waves in fluid dynamics. Osman et al. (2019b) investigated the non-autonomous Schrödinger-Hirota equation with power-law non-linearity using the unified method. Osman and Wazwaz (2019) studied (3 + 1)-dimensional Boiti-Leon-MannaPempinelli equation and analyzed the propagation and dynamical behaviors of the solutions with different free parameters. Liu et al. (2019) investigated the Hirota equation with variable coefficients. This method described the pulse propagation in inhomogeneous fibers more realistically than the other constant coefficient equation. Javid et al. (2019) applied the generalized unified method to analyze the thermophoretic motion equation. They analyzed the soliton-like thermophoresis of wrinkles in graphene sheet based on the Korteweg-de Vries $(\mathrm{KdV})$ equation and illustrated the solutions graphically. Osman et al. (2019c) applied the modified auxiliary equation method to investigate the complex wave structures related to the complex Ginzburg-Landau model. Lu et al. (2020) examined the Cahn-Hilliard equation to study the physical behaviors of the basic elements related to the phase decomposition of ( $\mathrm{Fe}-\mathrm{Cr}-\mathrm{Mo})$ and $(\mathrm{Fe}-\mathrm{Cr}-\mathrm{Cu})$ ternary alloys using both the analytical and numerical methods. Furthermore, they studied the relevant dynamical separation process.

Saratha et al. (2020) developed the FGHAM approach for solving non-linear fractional differential equations. The proposed method overcame the limitations of HAM by avoiding the iterative differentiation and integration requirements. Furthermore, the method also solved the limitations of usual Laplace-type integral transform ( $G$-transform).

The effectiveness of FGHAM motivated to study its application to tackle B-S equation in the European option pricing model. The merits of solving the B-S equation with FGHAM are clearly illustrated by identifying the convergence regions through the $h$-curve. The solutions thus obtained present an excellent agreement with the existing results.

The organization of this paper is as follows: Sect. 2 discusses the preliminaries of FGHAM for solving the B-S model, the basic definitions, and the fundamental results that are necessary for understanding the present analysis. Section 3 proposes an elaborate description of the new hybrid methodology to solve non-linear fractional differential equations. Section 4 justifies the reason for using FGHAM to solve the non-linear fractional B-S equation governing 
European option valuation under transaction costs. Section 5 presents an analysis of the non-linear generalized fractional B-S equation governing European option valuation under transaction costs. Section 6 presents an analysis of fractional B-S option pricing equation and also compares with Monte Carlo simulation. Section 7 validates the statistical analysis for the above numerical examples. Section 8 draws the concluding remarks and presents the suggestions for future research.

\section{Preliminaries}

This section discusses some basic definitions of fractional calculus used in this study.

Definition 2.1 The Riemann-Liovillie fractional integrals (Samko et al. 1993) of the left and right sides are defined for any function $\phi(x) \in L_{1}(a, b)$ as:

$$
\begin{aligned}
& \left(I_{a+}^{\alpha} \phi\right)(x)=\frac{1}{\Gamma(\alpha)} \int_{a}^{\infty}(x-t)^{\alpha-1} \phi(t) \mathrm{d} t, \quad x>a . \\
& \left(I_{b-}^{\alpha} \phi\right)(x)=\frac{1}{\Gamma(\alpha)} \int_{-\infty}^{b}(t-x)^{\alpha-1} \phi(t) \mathrm{d} t, \quad x<b .
\end{aligned}
$$

Definition 2.2 The Riemann integral on the half axis (Samko et al. 1993) subjects to a variable limit which can be expressed as:

$$
\left(I_{0+}^{\alpha} \phi\right)(x)=\frac{1}{\Gamma(\alpha)} \int_{0}^{x}(x-t)^{\alpha-1} \phi(t) \mathrm{d} t, \quad 0<x<\infty .
$$

Definition 2.3 The left- and right-handed Riemann-Liouville fractional derivatives (Samko et al. 1993) of order $\alpha, 0<\alpha<1$, in the interval $[a, b]$ are defined as:

$$
\begin{aligned}
& \left(D_{a+}^{\alpha} f\right)(x)=\frac{1}{\Gamma(1-\alpha)} \frac{\mathrm{d}}{\mathrm{d} x} \int_{a}^{x}(x-t)^{-\alpha} f(t) \mathrm{d} t . \\
& \left(D_{b-}^{\alpha} f\right)(x)=\frac{1}{\Gamma(1-\alpha)} \frac{\mathrm{d}}{\mathrm{d} x} \int_{x}^{b}(t-x)^{-\alpha} f(t) \mathrm{d} t .
\end{aligned}
$$

Definition 2.4 The Caputo fractional derivative of order $\alpha$ is defined as:

$$
D_{a}^{\alpha} f(x)=\frac{1}{\Gamma(m-\alpha)} \int_{a}^{x} \frac{f^{(m)} \xi}{(x-\xi)^{\alpha-m+1}} d \xi,
$$

where $m-1<\alpha \leq m, m \in N$.

Definition 2.5 The Mittag-Leffler function, which is a generalization of the exponential function, is defined as:

$$
E_{\alpha}(z)=\sum_{n=0}^{\infty} \frac{z^{n}}{\Gamma(\alpha n+1)},
$$

where $\alpha \in C, R(\alpha)>0$.

Definition 2.6 The continuous function $f: R \rightarrow R, t \rightarrow f(t)$ has a fractional derivative of order $k \alpha$. For any positive integer $k$ and for any $\alpha, 0<\alpha<1$, the Taylor series of fractional order is given by:

$$
f(t+h)=\sum_{k=0}^{\infty} \frac{h^{\alpha k}}{(\alpha k) !} f^{(\alpha k)}(t), \quad 0<\alpha<1,
$$


where $\Gamma(1+\alpha k)=(\alpha k)$.

Lemma 2.1 Let $f(t)$ be a continuous function. Then, the solution $y(t), y(0)=0$ is given by:

$$
\begin{aligned}
y & =\int_{0}^{t} f(\xi)(\mathrm{d} \xi)^{\alpha} \\
& =\alpha \int_{0}^{t}(t-\xi)^{(\alpha-1)} f(\xi) d \xi, \quad 0<\alpha<1 .
\end{aligned}
$$

\subsection{Fractional generalized integral transform (fractional G-transform)}

This section introduces the fractional $G$-transform and discusses some of the properties proposed by Saratha et al. (2020).

Definition 2.7 Let $g(t)$ be any time-domain function defined for $t>0$. Then, the fractional $G$-transform of order $\alpha$ of $g(t)$ is denoted by $G_{\alpha}[g(t)]$ and is defined as:

$$
\begin{aligned}
G_{\alpha}[g(t)] & =H_{\alpha}[u]=u^{p+1} \int_{0}^{\infty} g(u t) E_{\alpha}(-t)^{\alpha}(\mathrm{d} t)^{\alpha} \\
& =u^{p-\alpha+1} \int_{0}^{\infty} g(t) E_{\alpha}\left(\frac{-t}{u}\right)^{\alpha}(\mathrm{d} t)^{\alpha} \\
& =\lim _{M \rightarrow \infty} u^{p-\alpha+1} \int_{0}^{M} g(t) E_{\alpha}\left(\frac{-t}{u}\right)^{\alpha}(\mathrm{d} t)^{\alpha},
\end{aligned}
$$

where $E_{\alpha}$ is the Mittag-Leffler function.

The fractional $G$-transform satisfies the following properties:

Theorem 2.1 (Duality in fractional G-transform)

If the fractional-order Laplace Transform of a function $g(t)$ is $L_{\alpha}\{g(t)\}=F_{\alpha}(s)$, then the fractional G-transform of order $\alpha$ of $g(t)$ is:

$$
G_{\alpha}[g(t)]=H_{\alpha}(u)=u^{p-\alpha+1} F_{\alpha}\left(\frac{1}{u}\right) .
$$

Theorem 2.2 (Scaling property) If $G_{\alpha}[g(t)]=H_{\alpha}(u)$, then:

$$
G_{\alpha}[g(a t)]=\frac{1}{a^{\alpha}} H_{\alpha}\left[\frac{u}{a}\right],
$$

where a is a non-zero constant.

Theorem 2.3 If $G_{\alpha}[g(t)]=H_{\alpha}(u)$, then:

$$
G_{\alpha}[g(t-b)]=E_{\alpha}\left(\frac{-b}{u}\right)^{\alpha} H_{\alpha}(u) .
$$

Theorem 2.4 If $G_{\alpha}[g(t)]=H_{\alpha}(u)$, then:

$$
G_{\alpha}\left[E_{\alpha}\left(a^{\alpha} t^{\alpha}\right) g(t)\right]=\left(\frac{1}{1-a u^{2}}\right)^{\alpha} H_{\alpha}\left(\frac{u}{1-a u}\right) .
$$

The systematic procedure for the FGHAM (Saratha et al. 2020) is given in the next section. 


\section{Fractional generalized homotopy analysis method (FGHAM)}

Consider a fractional time non-linear partial differential equation with the following initial condition:

$$
D^{\alpha} v(x, t)+R v(x, t)+N v(x, t)=g(x, t), \quad v(x, 0)=f(x),
$$

where $D^{\alpha}$ is the fractional differential operator $D^{\alpha}=\frac{\partial^{\alpha}}{\partial t^{\alpha}}, R$ is the linear differential operator ,$N$ is the non-linear differential operator, and $g(x, t)$ is the source term.

The following systematic procedure steps are used to solve the non-linear fractional differential equations:

Step 1: Using fractional $G$-transform, Eq. (3.1) is transformed to:

$$
G_{\alpha}\left[D^{\alpha} v(x, t)\right]+G_{\alpha}[R v(x, t)]+G_{\alpha}[N v(x, t)]=G_{\alpha}[g(x, t)] .
$$

Step 2: Applying the derivative property of fractional $G$-transform, Eq. (3.2) is expressed as:

$$
G_{\alpha}[v(x, t)]-u^{p+1} v_{0}(x, t)+u^{\alpha}\left(G_{\alpha}[R v(x, t)]+G_{\alpha}[N v(x, t)]-G[g(x, t)]\right)=0 .
$$

Step 3: Decomposing the non-linear terms in Eq. (3.3), the following homotopy is constructed:

$$
(1-s) G_{\alpha}\left[\phi(x, t ; s)-v_{0}(x, t)\right]=h s H(x, t) N[\phi(x, t ; s)],
$$

where $s \in[0,1]$ is an embedding parameter and $\phi(x, t ; s)$ is a real function of $x, t$, and $s$, while $h$ is a non-zero auxiliary parameter, $H(x, t) \neq 0$ is an auxiliary function, $v_{0}(x, t)$ is an initial guess of $v(x, t)$, and $\phi(x, t ; s)$ is an unknown function. Equation (3.4) is called the zeroth-order deformation equation.

In (3.4), if $s=0$ and $s=1$, then $\phi(x, t, 0)=v_{0}(x, t)$ and $\phi(x, t, 1)=v(x, t)$, respectively. If $s \in[0,1]$, then the solution is transferred from $v_{0}(x, t)$ to $v(x, t)$.

Step 4: Deriving the $n^{t h}$-order deformation equation in (3.5) as in (Liao 1992):

$$
G_{\alpha}\left[v_{n}(x, t)-\chi_{n} v_{n-1}(x, t)\right]=h H(x, t) R_{n}\left(\mathbf{v}_{\mathbf{n}-1}(x, t)\right) .
$$

Step 5: Using the Inverse $G$-transform on both the sides of Eq. (3.5), the following equation is obtained:

$$
v_{n}(x, t)=\chi_{n} v_{n-1}(x, t)+h G_{\alpha}^{-1}\left[H(x, t) R_{n}\left(\mathbf{v}_{\mathbf{n}-1}, x, t\right)\right],
$$

where

$$
\begin{aligned}
R_{n}\left(\mathbf{v}_{\mathbf{n}-1}, x, t\right)= & G_{\alpha}[v(x, t)]-u^{p+1}\left(1-\chi_{n}\right) v_{0}(x, t) \\
& +u^{\alpha}\left(G_{\alpha}[R v(x, t)]+G[N v(x, t)]-G_{\alpha}[g(x, t)]\right) .
\end{aligned}
$$

and

$$
\chi_{n}= \begin{cases}0 & n \leq 1 \\ 1 & n>1\end{cases}
$$

Step 6: The following solution is obtained:

$$
v(x, t)=v_{0}(x, t)+\sum_{n=1}^{\infty} v_{n}(x, t) .
$$




\subsection{Error calculation and convergence of FGHAM}

It is essential to evaluate the convergence of the series solution obtained in Eq. (3.8) by FGHAM as in Bagyalakshmi et al. (2016). The approximate solution of (3.1) is obtained as $v_{\text {app }(k)}(x, t)=\sum_{n=0}^{k} v_{n}(x, t)$ from (3.8) by truncating the terms for $n=k+1, k+2, \ldots \infty$. Then, the exact solution of Eq. (3.1) is represented as:

$$
v(x, t)=v_{\text {app }(k)}(x, t)+e v_{k}(x, t),
$$

where $e v_{k}(x, t)$ is the error function. Generally, the absolute error is defined as $e v_{k}(x, t)=$ $\left|v(x, t)-v_{\operatorname{app}(k)}(x, t)\right|$. To establish convergence of equation (3.8), it is necessary to show that the sequence $E v_{k}(x, t)$ is a convergent sequence. Since the sequence is bounded below, it is sufficient to prove that the sequence $E v_{k}(x, t)$ is monotonically decreasing. As such, the convergence criteria are $\frac{E v_{p}(x, t)}{E v_{k}(x, t)}<1$ for $k<p$.

Using the following algorithm, convergence of the iterative solution $v_{\mathrm{app}(k)}(x, t)$ to the exact solution $v(x, t)$ is shown below:

- Compute $v_{\text {app }(k)}(x, t)$;

- Compute $v_{\mathrm{app}(p)}(x, t)$, for $k \leq p$;

- Define $E v_{k}(x, t)=\left|v(x, t)-v_{\text {app }(k)}(x, t)\right| E v_{p}(x, t)=\left|v(x, t)-v_{\operatorname{app}(p)}(x, t)\right|$ for some $x$ and $t$;

- If $E v_{k}(x, t) \geq E v_{p}(x, t)$, then $v_{\mathrm{app}(k)}(x, t)$ converges to the exact solution $v(x, t)$, when $k \rightarrow \infty$

The following section solves the fractional B-S equation and generalized fractional B-S equation using FGHAM. The obtained solutions are compared with those of the exact solution along with statistical validation. The results indicate an excellent agreement with some existing methods.

\section{Fractional Black-Scholes equation}

Considering the fractional B-S equation:

$$
\frac{\partial^{\alpha} v}{\partial t^{\alpha}}=\frac{\partial^{2} v}{\partial x^{2}}+(k-1) \frac{\partial v}{\partial x}-k v, \quad 0<\alpha \leq 1,
$$

with the initial condition: $v(x, 0)=\max \left(\mathrm{e}^{x}-1,0\right)$.

Equation (4.1) contains parameter $k=\frac{2 r}{\sigma^{2}}$, where $\mathrm{k}$ represents a balance between the interest rate and variability of stock returns, with the dimensionless time to expiry $\frac{\sigma^{2}}{2 T}$. The other four dimensionless parameters are the exercise price $E$, expiry $T$, volatility of the underlying asset $\sigma^{2}$, and risk-free interest rate $r$ as in the original problem. Applying the fractional $G$-transform on both the sides of Eq. (4.1):

$$
\begin{aligned}
& G_{\alpha}\left[\frac{\partial^{\alpha} v(x, t)}{\partial t^{\alpha}}\right]=G_{\alpha}\left[\frac{\partial^{2} v(x, t)}{\partial x^{2}}+(k-1) \frac{\partial v(x, t)}{\partial x}-k v(x, t)\right] \\
& \frac{1}{u^{\alpha}} G_{\alpha}[v(x, t)]-\frac{1}{u^{\alpha-1}} v(x, 0) u^{p}=G_{\alpha}\left[\frac{\partial^{2} v(x, t)}{\partial x^{2}}+(k-1) \frac{\partial v(x, t)}{\partial x}-k v(x, t)\right] \\
& G_{\alpha}[v(x, t)]-u^{p+1} v(x, 0)-u^{\alpha} G_{\alpha}\left[\frac{\partial^{2} v(x, t)}{\partial x^{2}}+(k-1) \frac{\partial v(x, t)}{\partial x}-k v(x, t)\right]=0 .
\end{aligned}
$$


Applying FGHAM:

$$
v_{n}(x, t)=\chi_{n} v_{n-1}(x, t)+h G_{\alpha}^{-1}\left[R_{n}\left(\mathbf{v}_{\mathbf{n}-1}, x, t\right)\right],
$$

where

$$
\begin{aligned}
R_{n}\left[\mathbf{v}_{\mathbf{n}-1}, x, t\right]= & G_{\alpha}\left[v_{n-1}(x, t)\right]-\left(1-\chi_{n}\right) u^{p+1} v(x, 0) \\
& -u^{\alpha} G_{\alpha}\left[\frac{\partial^{2} v_{n-1}(x, t)}{\partial x^{2}}+(k-1) \frac{\partial v_{n-1}(x, t)}{\partial x}-k v_{n-1}(x, t)\right] .
\end{aligned}
$$

Solving the above equation for $n=1,2,3, \ldots$ :

$$
\begin{aligned}
v_{0}(x, t)= & \max \left(\mathrm{e}^{x}-1,0\right) \\
v_{1}(x, t)= & -h k \frac{t^{\alpha}}{\Gamma(\alpha+1)}\left[\max \left(\mathrm{e}^{x}, 0\right)-\max \left(\mathrm{e}^{x}-1,0\right)\right] \\
v_{2}(x, t)= & -h(h+1) k \frac{t^{\alpha}}{\Gamma(\alpha+1)}\left[\max \left(\mathrm{e}^{x}, 0\right)-\max \left(\mathrm{e}^{x}-1,0\right)\right] \\
& -h^{2} k^{2} \frac{t^{2 \alpha}}{\Gamma(2 \alpha+1)}\left[\max \left(\mathrm{e}^{x}, 0\right)-\max \left(\mathrm{e}^{x}-1,0\right)\right] \\
v_{3}(x, t)= & (1+h) v_{2}(x, t)-h^{2}(h+1) k^{2} \frac{t^{2 \alpha}}{\Gamma(2 \alpha+1)}\left[\max \left(\mathrm{e}^{x}, 0\right)-\max \left(\mathrm{e}^{x}-1,0\right)\right] \\
& -h^{3} k^{3} \frac{t^{3 \alpha}}{\Gamma(3 \alpha+1)}\left[\max \left(\mathrm{e}^{x}, 0\right)-\max \left(\mathrm{e}^{x}-1,0\right)\right] .
\end{aligned}
$$

Similarly, $v_{4}, v_{5}, \ldots$ are estimated and the series solution is obtained, that is:

$$
\begin{aligned}
& v(x, t)=\sum_{n=0}^{\infty} v_{n}(x, t) \\
& v(x, t)=v_{0}(x, t)+v_{1}(x, t)+v_{2}(x, t)+v_{3}(x, t)+\cdots
\end{aligned}
$$

If $h=-1$, Eq. (4.2) can be expressed as:

$$
v(x, t)=\max \left(\mathrm{e}^{x}-1,0\right) E_{\alpha}\left(-k t^{\alpha}\right)+\max \left(\mathrm{e}^{x}, 0\right)\left(1-E_{\alpha}\left(-k t^{\alpha}\right)\right),
$$

where $E_{\alpha}$ represents the Mittag-Leffler function.

The series solution of the fractional time equation (4.1) obtained in terms of the MittagLeffler function in Eq. (4.3) approaches the exact solution shown in Eq. (4.4), when $\alpha=1$ $\div$ :

$$
v(x, t)=\max \left(\mathrm{e}^{x}-1,0\right) \mathrm{e}^{-k t}+\max \left(\mathrm{e}^{x}, 0\right)\left(1-\mathrm{e}^{-k t}\right) .
$$

Case 1. Consider the Vanilla call option (Company et al. 2008) with parameter $\sigma=0.2$, $r=0.04, \alpha=1, \tau=0.5$ year, then $k=2$.

The solution of Eq. (4.4) is:

$$
v(x, t)=\max \left(\mathrm{e}^{x}-1,0\right) \mathrm{e}^{-2 t}+\max \left(\mathrm{e}^{x}, 0\right)\left(1-\mathrm{e}^{-2 t}\right) .
$$

Equation (4.5) is the exact solution of the standard B-S equation.

Table 1 shows the absolute errors subject to some particular points $\alpha=1$ and $x=0.5$. This proves the convergence of the series solution of (4.1). Figure 1 depicts a comparison of the absolute errors for the different sequences of partial sums. 
Table 1 Absolute errors for the Black-Scholes equation

\begin{tabular}{|c|c|c|c|}
\hline $\begin{array}{l}t \\
0\end{array}$ & $\begin{array}{l}E v_{3}(x, t) \\
0 .\end{array}$ & $\begin{array}{l}E v_{4}(x, t) \\
0 .\end{array}$ & $\begin{array}{l}E v_{5}(x, t) \\
0 .\end{array}$ \\
\hline 0.1 & -0.0000640864 & $2.58026 \times 10^{-} 6$ & $-8.64113 * 10^{-} 8$ \\
\hline 0.2 & -0.000986713 & 0.000079954 & $-5.37937 * 10^{-} 6$ \\
\hline 0.3 & -0.00481164 & 0.000588364 & -0.0000596361 \\
\hline 0.4 & -0.0146623 & 0.00240437 & -0.000326297 \\
\hline 0.5 & -0.0345461 & 0.00712056 & -0.00121277 \\
\hline 0.6 & -0.0691942 & 0.0172058 & -0.00353021 \\
\hline 0.7 & -0.12393 & 0.0361364 & -0.0086823 \\
\hline 0.8 & -0.204563 & 0.0685035 & -0.0188779 \\
\hline 0.9 & -0.317299 & 0.120101 & -0.0373629 \\
\hline 1 & -0.468669 & 0.197998 & -0.0686686 \\
\hline
\end{tabular}

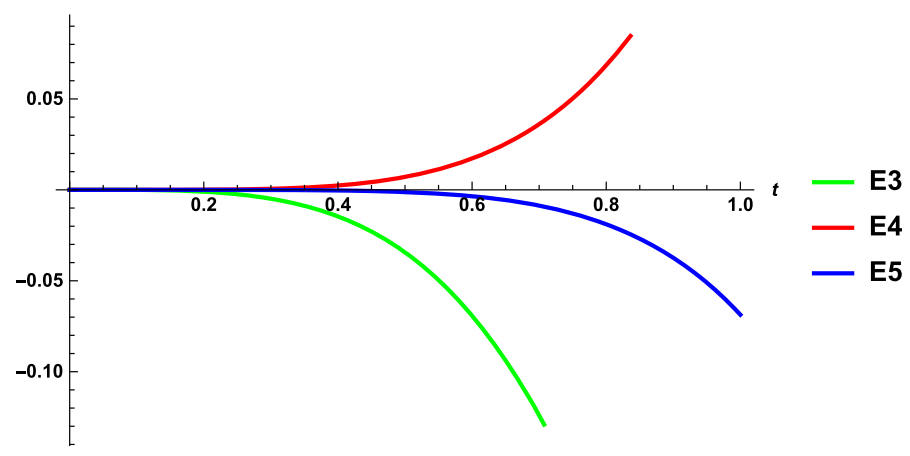

Fig. 1 A comparison of absolute errors between FGHAM and exact solution

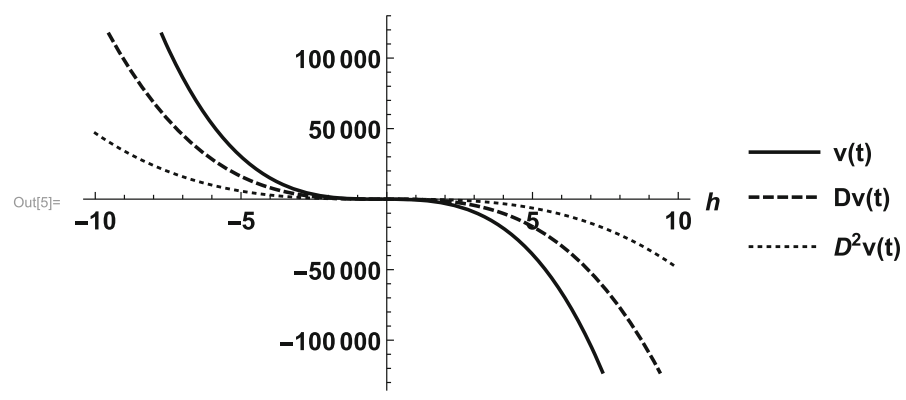

Fig. 2 The $h$-curve of the solution for the B-S equation

\subsection{Results and discussion}

The convergence region is obtained using the $h$-curve. Figure 2 shows the convergence region of Eq. (4.1) between -2 and 1. Figure 3 indicates that the FGHAM results almost coincide with those of HAM (Kumar et al. 2014), HPM [Kumar et al. (2014)], MFDTM [Ravi Kanth and Aruna (2016)], RPS [Jena and Chakraverty (2019)], CFADM [Yavuz and Ozdemir (2018)], and exact solution $v(x, t)$ of the $\mathrm{B}-\mathrm{S}$ equation. Figure 4 illustrates the solution of 


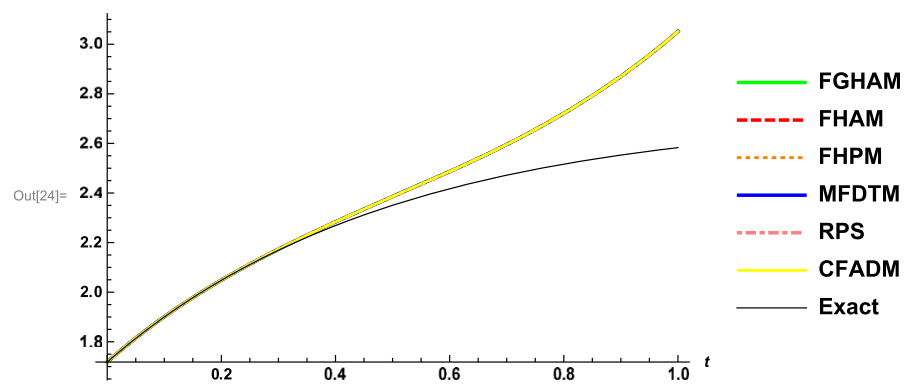

Fig. 3 Comparison among the results of FGHAM, HAM, HPM, MFDTM, RPS, CFADM, and the exact solution for the $\mathrm{B}-\mathrm{S}$ equation using the fractional parameter $\alpha=1$

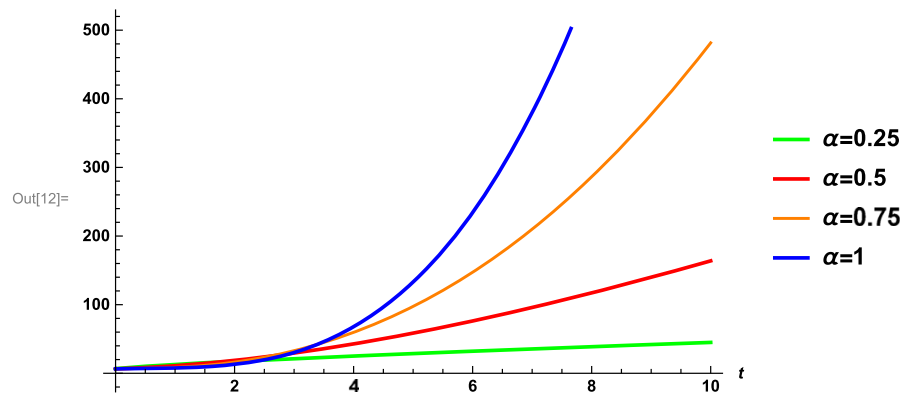

Fig. 4 A graphical illustration of the solution for the B-S equation using various settings of fractional parameter $\alpha=0.25,0.5,0.75,1$

the B-S equation with the various settings of the fractional parameter $\alpha=0.25,0.5,0.75,1$, respectively.

Figure 5 depicts the financial pricing derivatives subjects to the different settings of the fractional parameter $\alpha=0.25,0.5,0.75$ and 1 .

Table 2 provides the pricing option derivatives using the fractional parameter $\alpha=1$, which depicts a good agreement with the results of FGHAM, exact solution, RPS, and CFADM, respectively.

Table 3 and 4 provide the pricing option derivatives using the fractional parameter $\alpha=$ $0.75, \alpha=0.5$, and depict a good agreement with the results of FGHAM, MFDTM, RPS, and CFADM, respectively.

Case 2. Consider the Vanilla call option (Company et al. 2008) with parameter $\sigma=0.2$, $r=0.01, \alpha=1, \tau=1$ year, then $k=5$.

The solution of equation (4.6) is obtained as:

$$
v(x, t)=\max \left(\mathrm{e}^{x}-1,0\right) \mathrm{e}^{-5 t}+\max \left(\mathrm{e}^{x}, 0\right)\left(1-\mathrm{e}^{-5 t}\right) .
$$

Equation (4.6) is the exact solution for the given equation.

Table 5 shows the absolute errors with respect to some particular points $\alpha=1$ and $x=1$. This proves the convergence of the series solution of (4.1). Figure 6 shows a comparison of the approximate absolute errors subject to the different sequences of partial sums. 


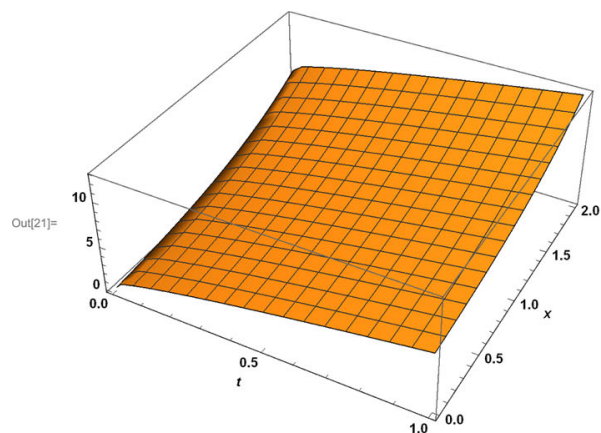

(a)

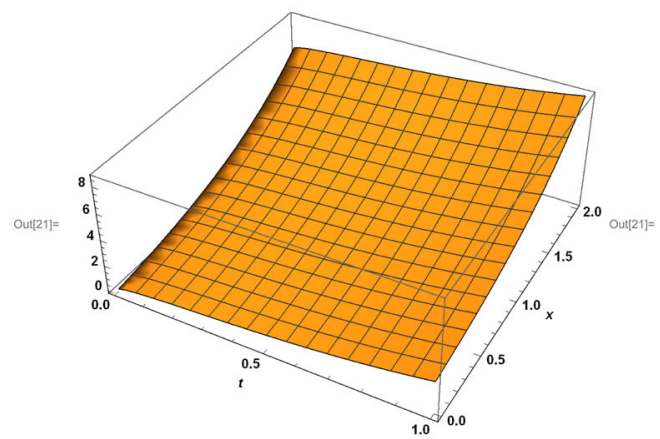

(c)

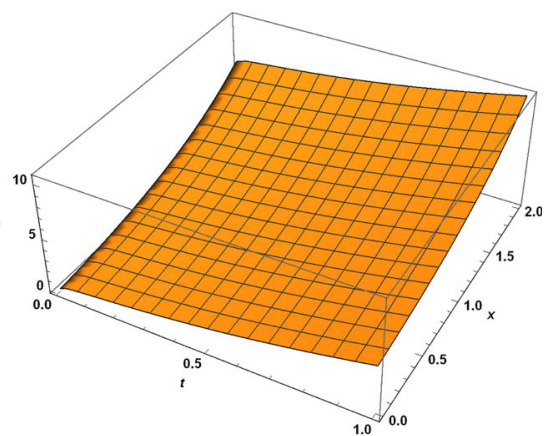

(b)

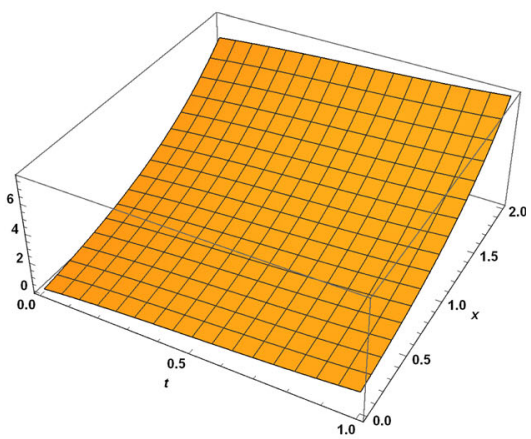

(d)

Fig. 5 The solution for Black-Scholes equation subject to various settings of fractional parameter $\alpha=$ $0.25,0.5,0.75,1$ and auxiliary parameter $h=-1$ are shown in $\mathbf{a}-\mathbf{d}$, respectively

\subsection{Results and discussion}

The convergence region is obtained using the $h$-curve. Figure 7 shows that the convergence region of Eq. (4.1) is between -2 and 2. Figure 8 indicates that the FGHAM results almost coincide with those of HAM, HPM, MFDTM, RPS, CFADM, and the exact solution for the B-S equation. Figure 9 illustrates the solution for the B-S equation subject to the various settings of the fractional parameter $\alpha=0.25,0.5,0.75,1$,

Figures 10 depicts the financial pricing derivatives for the different settings of the fractional parameter $\alpha=0.25,0.5,0.75$ and 1 , respectively.

Table 6 provides the pricing option derivatives using fractional parameter $\alpha=1$, depicts a good agreement among the results of FGHAM, the exact solution, RPS, and CFADM respectively.

Tables 7 and 8 provide the pricing option derivatives using fractional parameter $\alpha=0.75$, $\alpha=0.5$, and depict a good agreement among the results of FGHAM, MFDTM, RPS, and CFADM, respectively.

\subsection{Non-local behavior of implied volatility}

The implied volatility, an important financial parameter, which plays a vital role in pricing option problem. Generally, due to the mathematical structure of the integer order B-S formula, 


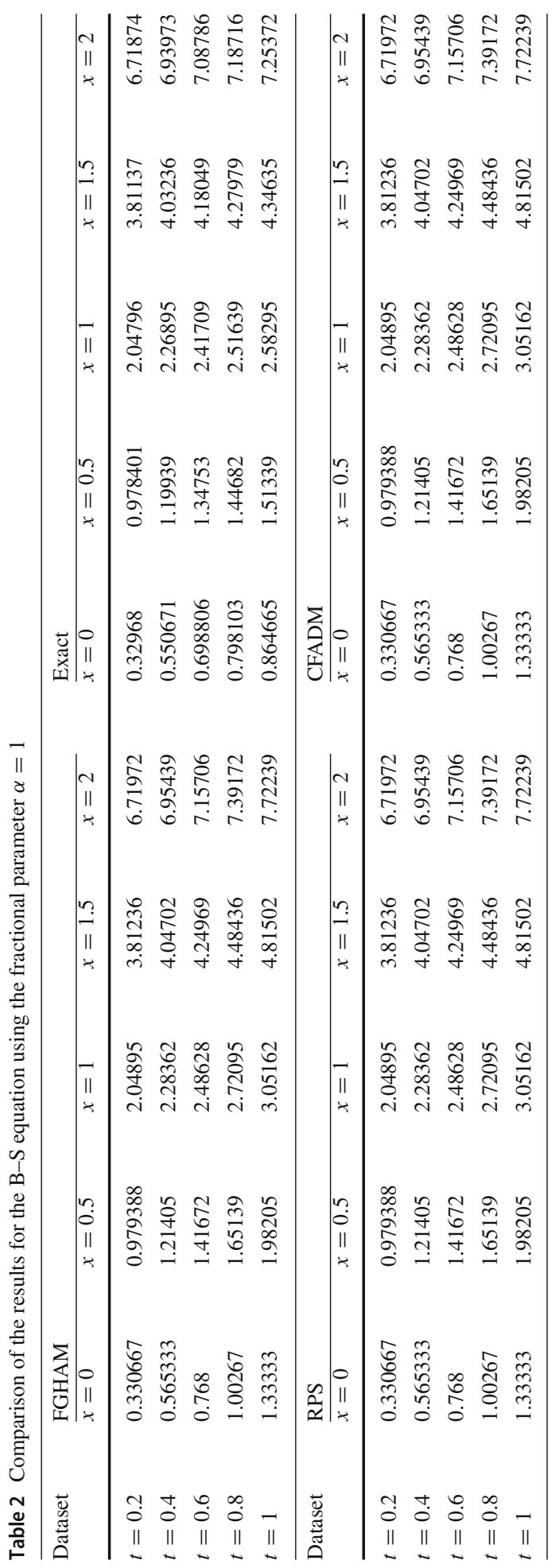




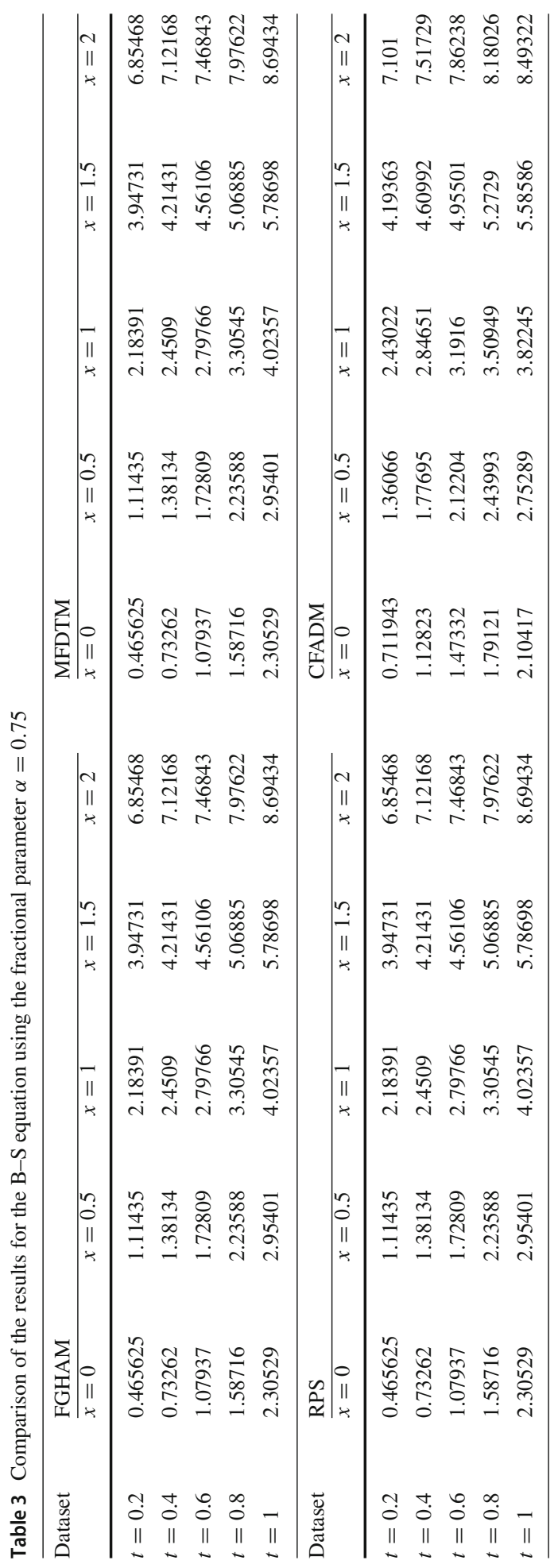




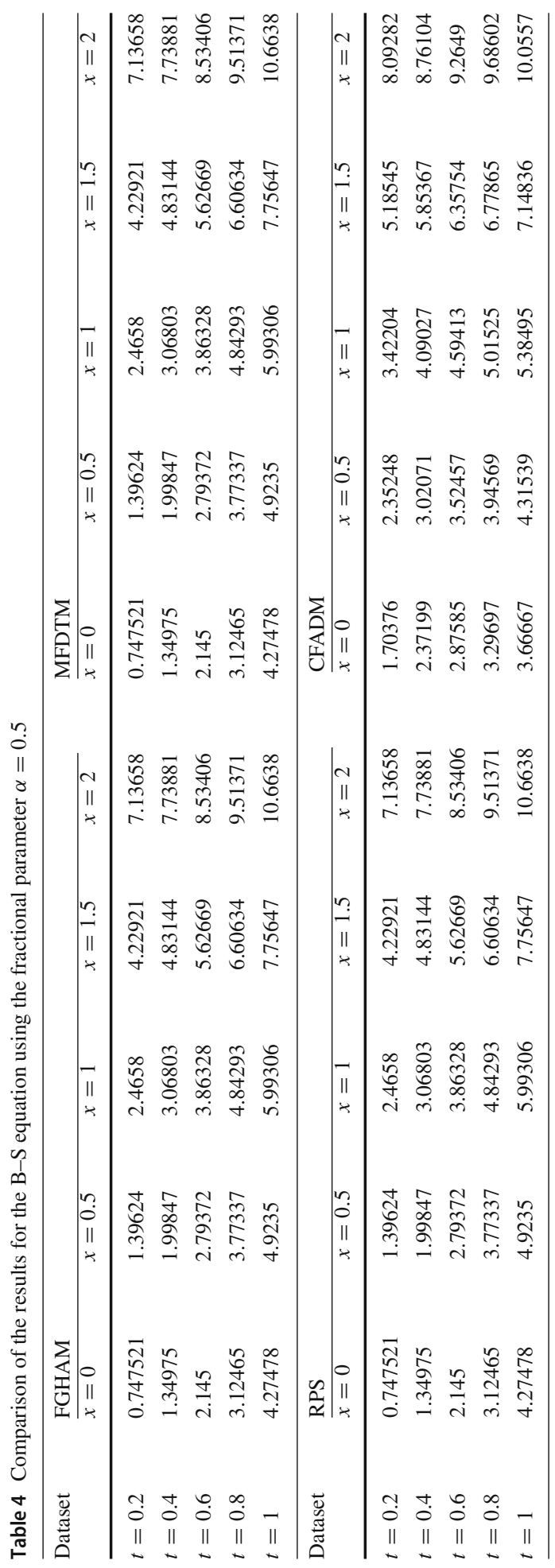




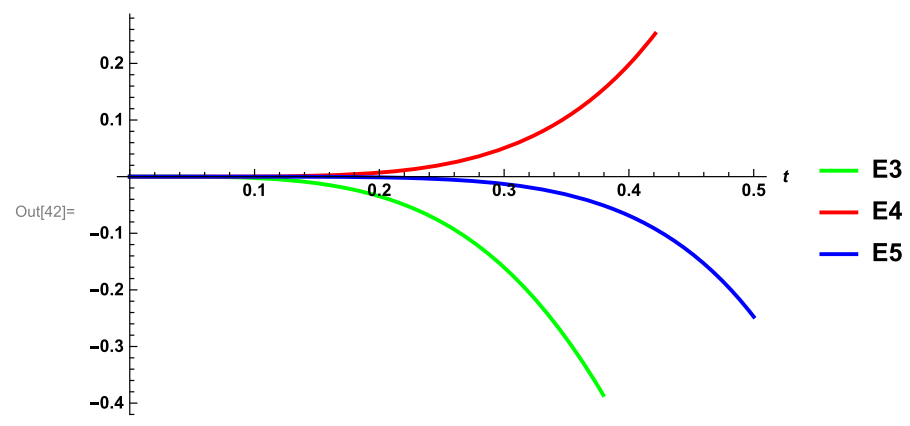

Fig. 6 A comparison of absolute errors between FGHAM and exact solution

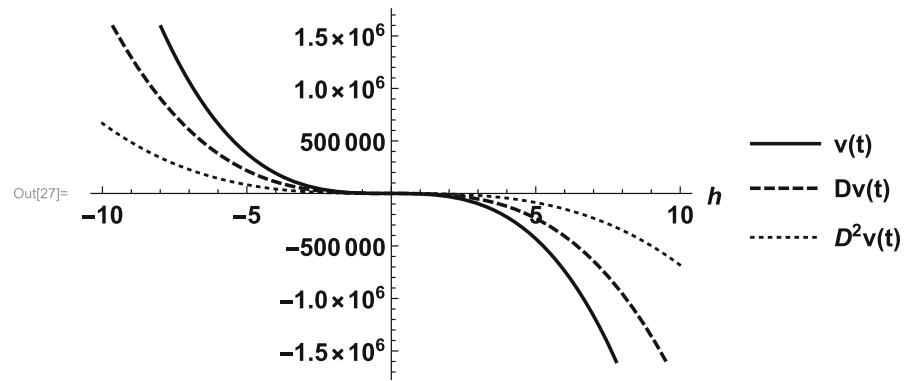

Fig. 7 The $h$-curve of the solution for the B-S equation

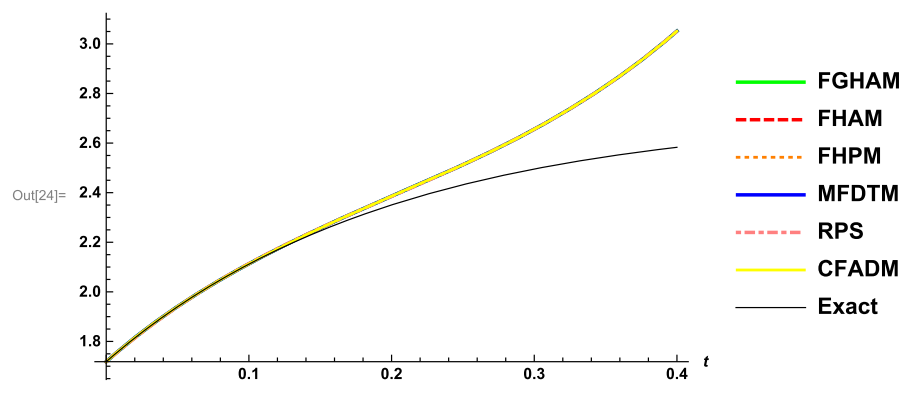

Fig. 8 Comparison among the results of FGHAM, HAM, HPM, MFDTM, RPS, CFADM, and the exact solution for the B-S equation using fractional parameter $\alpha=1$

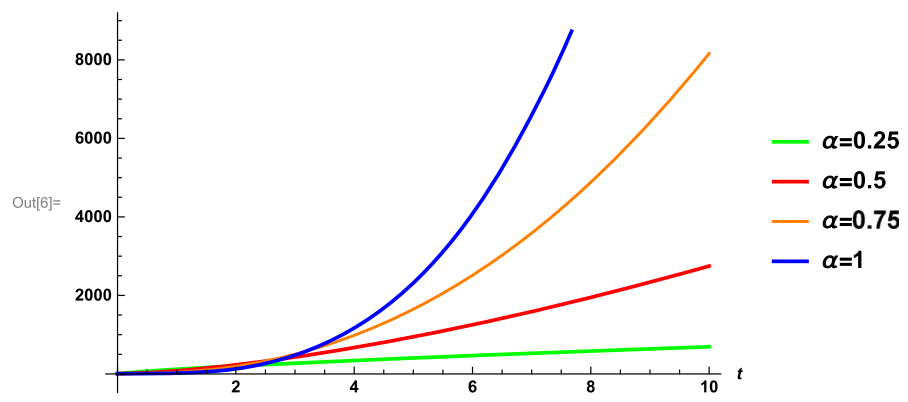

Fig. 9 A graphical illustration of the solution for the B-S equation subject to the various settings of the fractional parameter $\alpha=0.25,0.5,0.75,1$ 
Table 5 Absolute errors calculation for the $\mathrm{B}-\mathrm{S}$ equation

\begin{tabular}{llll}
\hline$t$ & $\begin{array}{l}E v_{3}(x, t) \\
0\end{array}$ & $\begin{array}{l}E v_{4}(x, t) \\
0 .\end{array}$ & $\begin{array}{l}E v_{5}(x, t) \\
0 .\end{array}$ \\
\hline 0.05 & -0.00015495 & $7.81068 \times 10^{-} 6$ & $-3.27342 \times 10^{-7}$ \\
0.1 & -0.00236399 & 0.000240174 & -0.000020243 \\
0.15 & -0.0114291 & 0.00175454 & -0.000222998 \\
0.20 & -0.0345461 & 0.00712056 & -0.00121277 \\
0.25 & -0.0807756 & 0.0209496 & -0.00448168 \\
0.30 & -0.16063 & 0.0503073 & -0.0129739 \\
0.35 & -0.285753 & 0.105035 & -0.0317411 \\
0.40 & -0.468669 & 0.197998 & -0.0686686 \\
0.45 & -0.722587 & 0.345284 & -0.135258 \\
0.50 & -1.06125 & 0.566353 & -0.24745 \\
\hline
\end{tabular}

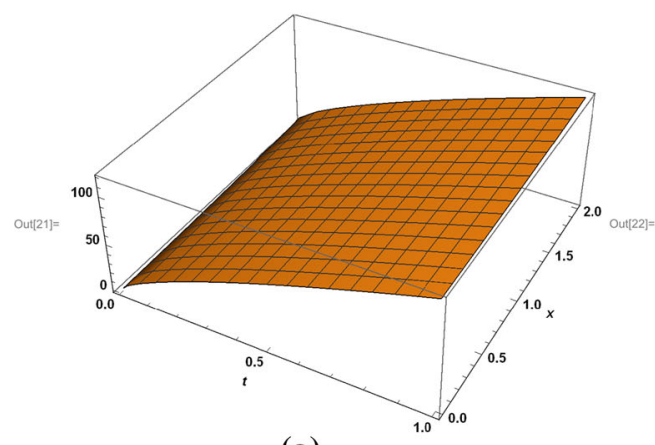

(a)

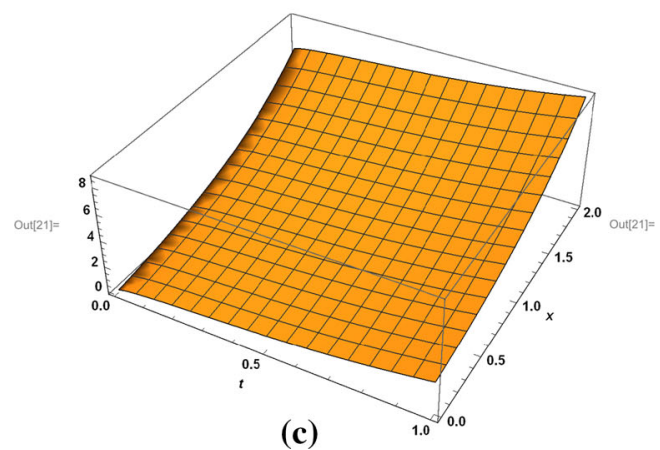

(c)

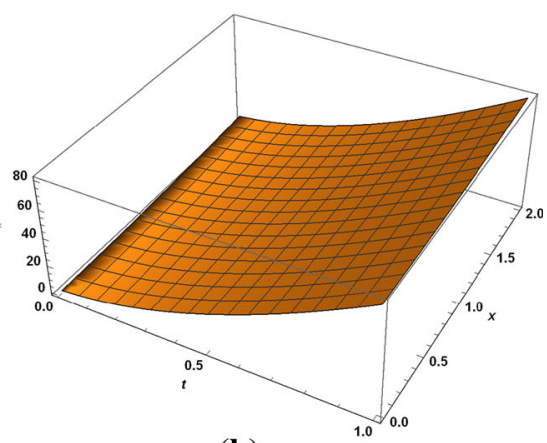

(b)

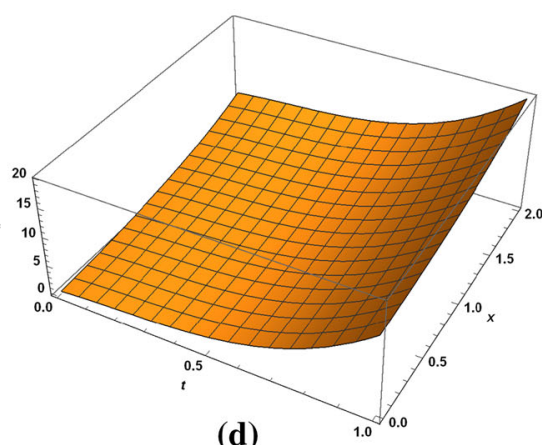

(d)

Fig. 10 The solution for the B-S equation subject to the various settings of the fractional parameter $\alpha=$ $0.25,0.5,0.75,1$ and the auxiliary parameter $h=-1$ are shown in $\mathbf{a}-\mathbf{d}$, respectively

the analytical expression to estimate the implied volatility cannot be obtained in the closed form. However, it is worth to mention that the presence of fractional parameter $\alpha$ in the fractional B-S mathematical model has an advantage of memory-less property. Thus, the non-local behavior of the implied volatility in terms of option can be analytically estimated in the closed form for the various values of the fractional parameter $\alpha$. 


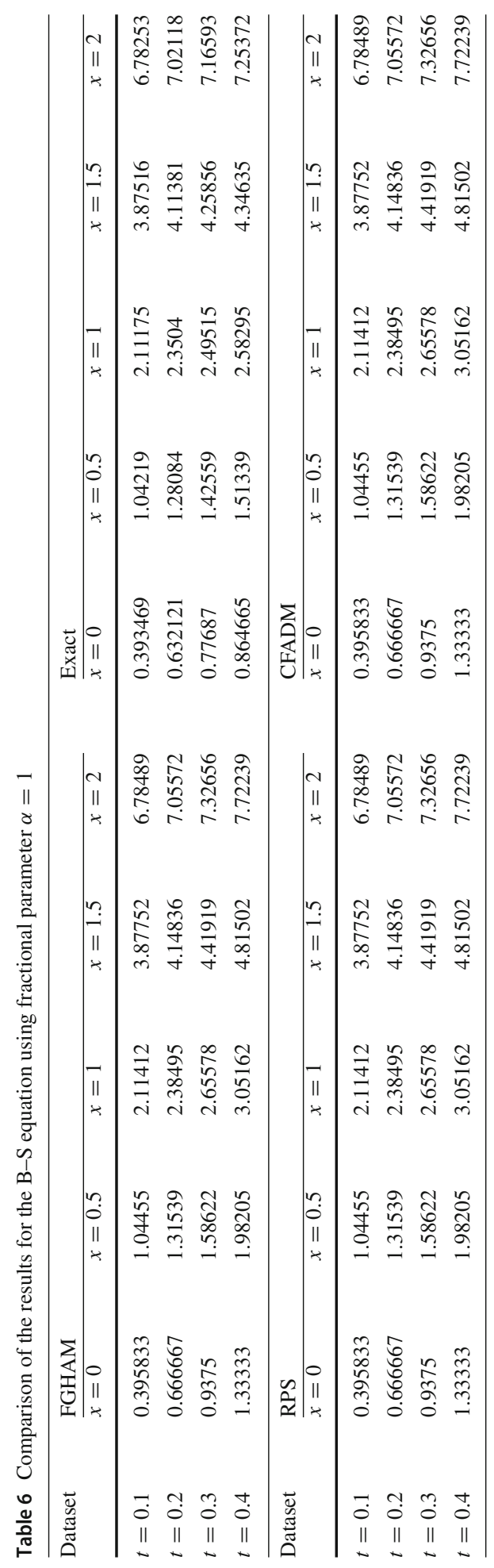




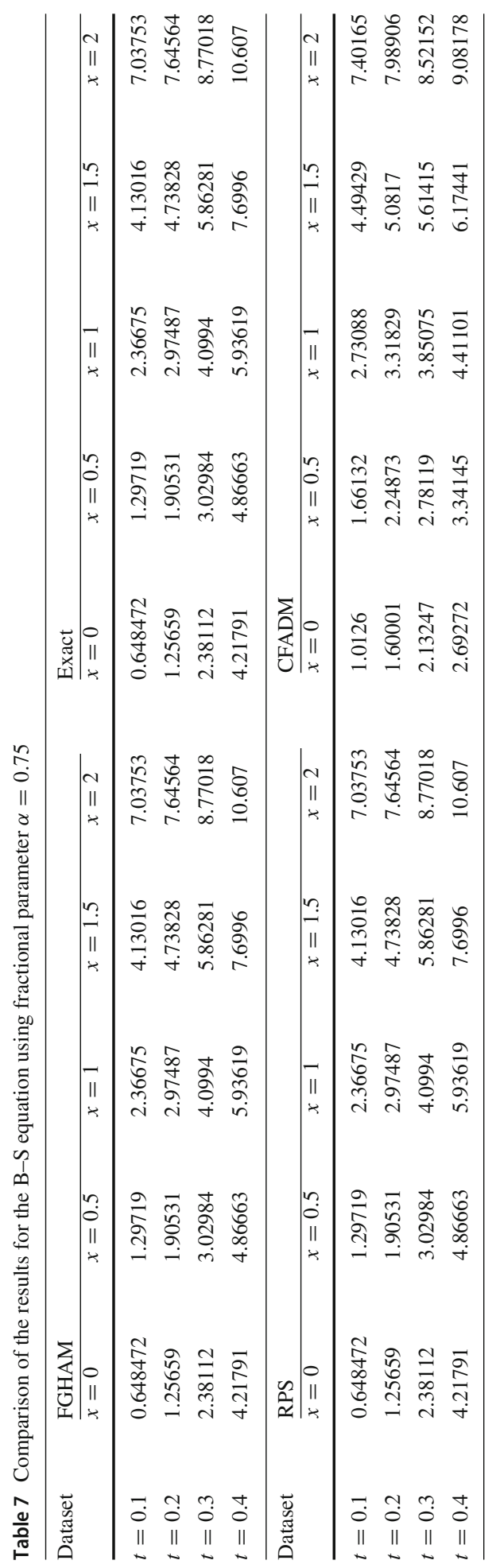




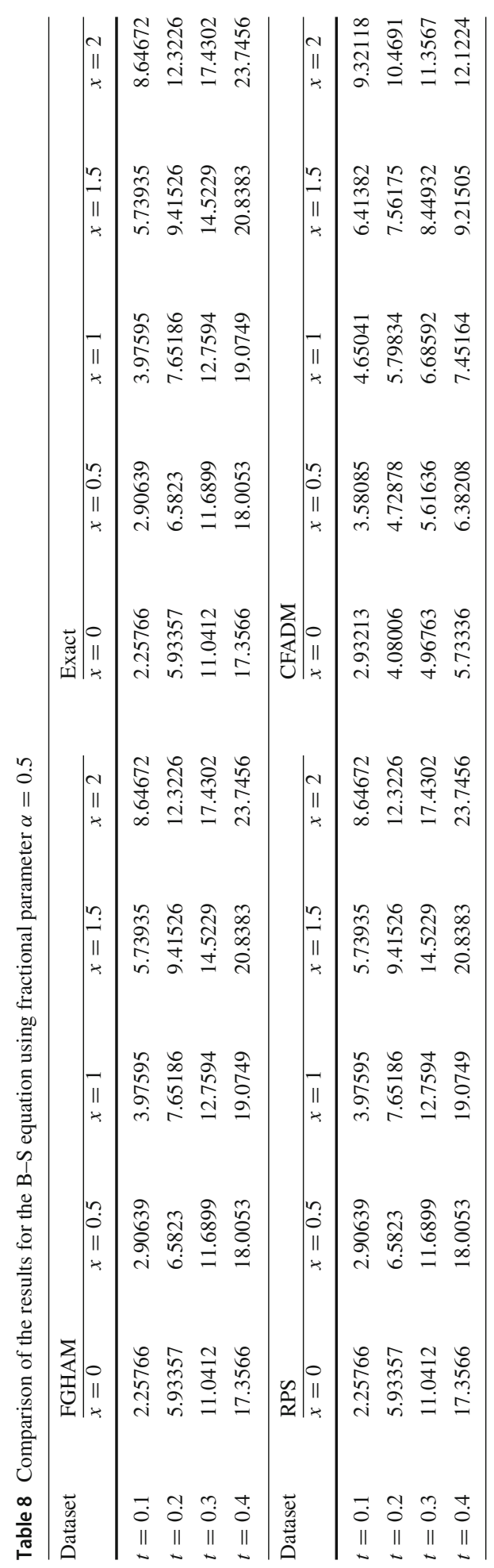


Table 9 Implied volatility for various values of $\alpha, k=2, r=0.04, \sigma=0.2$, and $t=0.5$

\begin{tabular}{lllll}
\hline Dataset & $\sigma_{0.25}$ & $\sigma_{0.5}$ & $\sigma_{0.75}$ & $\sigma_{1}=\sigma$ \\
\hline$x=5$ & 0.26959013221709927 & 0.25020190169869055 & 0.22534626071806207 & 0.19999999999999615 \\
$x=10$ & 0.2695901322167636 & 0.2502019016993923 & 0.22534626071782968 & 0.2 \\
$x=15$ & 0.269590132197852 & 0.2502019016920359 & 0.22534626079582623 & 0.20000000006328994 \\
$x=20$ & 0.26959013146068167 & 0.2502019010464151 & 0.22534625921403661 & 0.2 \\
$x=25$ & 0.269588858270559 & 0.25020118683230536 & 0.22534732629077037 & 0.19999896305091158 \\
$x=30$ & 0.26983459277301525 & 0.24979419077707743 & 0.2252233381567576 & 0.20039801034391733 \\
$\mathrm{x}=35$ & 0.2 & 0.2 & 0.17888543819998318 & 0.1932993161855452 \\
\hline
\end{tabular}

Table 10 Implied volatility $\sigma_{\alpha}$ for various values of $\alpha, k=5, r=0.01, \sigma=0.2$, and $t=1$

\begin{tabular}{lllll}
\hline Dataset & $\sigma_{0.25}$ & $\sigma_{0.5}$ & $\sigma_{0.75}$ & $\sigma_{1}=\sigma$ \\
\hline$x=5$ & 0.15148352991336414 & 0.14407514870762786 & 0.15247084850620074 & 0.1932455532033679 \\
$x=10$ & 0.15148352991267927 & 0.14407514870761357 & 0.15247084850689208 & 0.19324555320331422 \\
$x=15$ & 0.15148352981675964 & 0.1440751489405439 & 0.15247084831011348 & 0.19324555322044148 \\
$x=20$ & 0.15148353648258622 & 0.1440751628169402 & 0.15247083948762233 & 0.19324555276627591 \\
$x=25$ & 0.15147975638390557 & 0.14407393257075862 & 0.15247068853641338 & 0.19324594484458788 \\
$x=30$ & 0.1513339379792706 & 0.14319019692101542 & 0.1526270590592904 & 0.19327419053931703 \\
\hline
\end{tabular}

The implied volatility function $\sigma(x, t)$ with respect to the fractional parameter $\alpha$ is denoted by $\sigma_{\alpha}(x, t)$ and can be derived from (4.1) as shown below: (Dadachanji 2015).

$$
\sigma_{\alpha}(x, t)=\sqrt{\frac{2 r\left[\frac{\partial v(x, t)}{\partial x}-v(x, t)\right]}{\frac{\partial^{\alpha} v(x, t)}{\partial t^{\alpha}}-\frac{\partial^{2} v(x, t)}{\partial x^{2}}+\frac{\partial v(x, t)}{\partial x}}} .
$$

Using Eq. (4.7), the implied volatility $\sigma_{\alpha}$ is estimated for the various values of fractional parameter $\alpha$ and the results are shown in Tables 9 and 10. Even though the implied volatility is assumed to be a constant, the observation from the Tables 9 and 10 clearly reveals the pre-local change in the implied volatility. This result illustrates the importance of the fractional-order B-S model over the integer order in analyzing the non-local behavior of the implied volatility.

Even though the B-S model is considered with the constant volatility due to the presence of the fractional parameter $\alpha$, the non-local behavior of implied volatility $[\alpha \in(0,1)]$ is observed and the results are presented in Tables 9 and 10.

\section{Generalized fractional Black-Scholes equation}

Considering the following generalized B-S equation:

$$
\frac{\partial^{\alpha} v(x, t)}{\partial t^{\alpha}}+0.08(2+\sin x)^{2} x^{2} \frac{\partial^{2} v(x, t)}{\partial x^{2}}+0.06 x \frac{\partial v(x, t)}{\partial x}-0.06 v(x, t)=0,0<\alpha \leq 1,
$$

with the initial condition $v(x, 0)=\max \left(x-25 \mathrm{e}^{-0.06}, 0\right)$. 
Applying the fractional $G$-transform on both the sides of Eq. (5.1):

$$
\begin{aligned}
& G_{\alpha}\left[\frac{\partial^{\alpha} v(x, t)}{\partial t^{\alpha}}+0.08(2+\sin x)^{2} x^{2} \frac{\partial^{2} v(x, t)}{\partial x^{2}}+0.06 x \frac{\partial v(x, t)}{\partial x}-0.06 v(x, t)\right]=0 \\
& G_{\alpha}[v(x, t)]-u^{p+1} v(x, 0) \\
& \quad+u^{\alpha} G_{\alpha}\left[0.08(2+\sin x)^{2} x^{2} \frac{\partial^{2} v(x, t)}{\partial x^{2}}+0.06 x \frac{\partial v(x, t)}{\partial x}-0.06 v(x, t)\right]=0 .
\end{aligned}
$$

Applying FGHAM $\div$ :

$$
v_{n}(x, t)=\chi_{n} v_{n-1}(x, t)+h G_{\alpha}^{-1}\left[R_{n}\left(\mathbf{v}_{\mathbf{n}-1}, x, t\right)\right],
$$

where

$$
\begin{aligned}
R_{n}\left[\mathbf{v}_{\mathbf{n}-1}, x, t\right]= & G_{\alpha}\left[v_{n-1}(x, t)\right]-\left(1-\chi_{n}\right) u^{p+1} v(x, 0) \\
& +u^{\alpha} G_{\alpha}\left[0.08(2+\sin x)^{2} x^{2} \frac{\partial^{2} v(x, t)}{\partial x^{2}}+0.06 x \frac{\partial v(x, t)}{\partial x}-0.06 v(x, t)\right] .
\end{aligned}
$$

Solving the above equation for $n=1,2,3, \ldots$ :

$$
\begin{aligned}
v_{0}(x, t)= & \max \left(x-25 \mathrm{e}^{-0.06}, 0\right) \\
v_{1}(x, t)= & h x \frac{0.06 t^{\alpha}}{\Gamma(\alpha+1)}-h \max \left(x-25 \mathrm{e}^{-0.06}, 0\right) \frac{0.06 t^{\alpha}}{\Gamma(\alpha+1)} \\
v_{2}(x, t)= & h(h+1) \frac{0.06 t^{\alpha}}{\Gamma(\alpha+1)}\left[x-\max \left(x-25 \mathrm{e}^{-0.06}, 0\right)\right] \\
& -h^{2} \frac{(0.06)^{2} t^{2 \alpha}}{\Gamma(2 \alpha+1)}\left[x-\max \left(x-25 \mathrm{e}^{-0.06}, 0\right)\right] \\
v_{3}(x, t)= & (1+h) v_{2}(x, t)-h^{2}(h+1) \frac{(0.06)^{2} t^{2 \alpha}}{\Gamma(2 \alpha+1)}\left[x-\max \left(x-25 \mathrm{e}^{-0.06}, 0\right)\right] \\
& +h^{3} \frac{(0.06)^{3} t^{3 \alpha}}{\Gamma(3 \alpha+1)}\left[x-\max \left(x-25 \mathrm{e}^{-0.06}, 0\right)\right] .
\end{aligned}
$$

Similarly, $v_{4}, v_{5}, \ldots$ are estimated and the series solution is obtained as $\div$ :

$$
\begin{aligned}
& v(x, t)=\sum_{n=0}^{\infty} v_{n}(x, t) \\
& v(x, t)=v_{0}(x, t)+v_{1}(x, t)+v_{2}(x, t)+v_{3}(x, t)+\cdots
\end{aligned}
$$

If $h=-1$, Eq. (5.2) can be expressed as:

$$
v(x, t)=x\left(1-E_{\alpha}\left(-0.06 t^{\alpha}\right)\right)+\max \left(x-25 \mathrm{e}^{-0.06}, 0\right) E_{\alpha}\left(-0.06 t^{\alpha}\right),
$$

where $E_{\alpha}$ represents the Mittag-Leffler function.

The series solution of the fractional time equation (5.1) obtained in term of the MittagLeffler function in Eq. (5.3) approaches the exact solution as shown in Eq. (5.4), when $\alpha=1$ :

$$
v(x, t)=x\left(1-\mathrm{e}^{0.06 t}, 0\right)+\max \left(x-25 \mathrm{e}^{-0.06}, 0\right) \mathrm{e}^{-0.06 t} .
$$

Table 11 shows the absolute errors with respect to some particular points $\alpha=1$ and $x=5$. This proves the convergence of series solution of Eq. (5.1). Figure 11 depicts a comparison of the approximate absolute errors subject to the different sequence of the partial sums. 
Table 11 Absolute errors for the generalized B-S equation

\begin{tabular}{llll}
\hline$t$ & $E v_{3}(x, t)$ & $\begin{array}{l}E v_{4}(x, t) \\
0 .\end{array}$ & $\begin{array}{l}E v_{5}(x, t) \\
0 .\end{array}$ \\
\hline 2 & 0. & $-1.0579 * 10^{-} 6$ & $-2.10969 \times 10^{-} 8$ \\
4 & -0.0000442579 & -0.0000345516 & $-1.37401 \times 10^{-} 6$ \\
6 & -0.000725752 & -0.000267873 & -0.0000159304 \\
8 & -0.00376707 & -0.00115281 & -0.0000911278 \\
10 & -0.012212 & -0.003594 & -0.000354002 \\
12 & -0.030594 & -0.00913885 & -0.0010767 \\
14 & -0.0651261 & -0.0201917 & -0.00276619 \\
16 & -0.123915 & -0.0402552 & -0.0062813 \\
18 & -0.217202 & -0.0742026 & -0.0129806 \\
20 & -0.357638 & -0.128585 & -0.0249046 \\
\hline
\end{tabular}

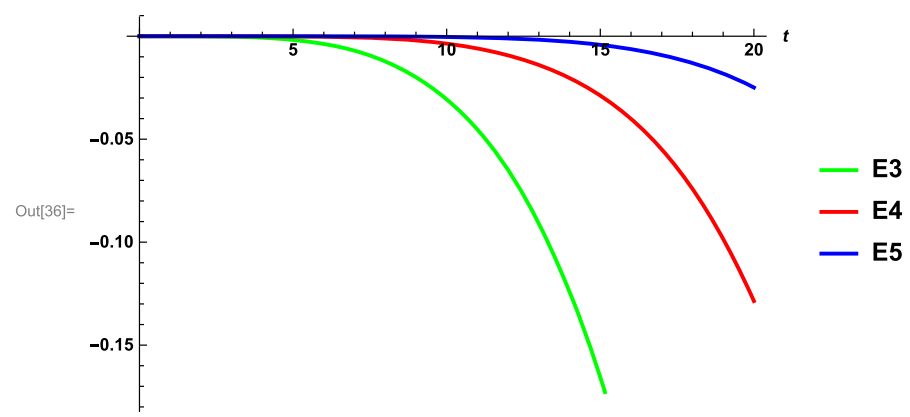

Fig. 11 A comparison of absolute errors between FGHAM and the exact solution

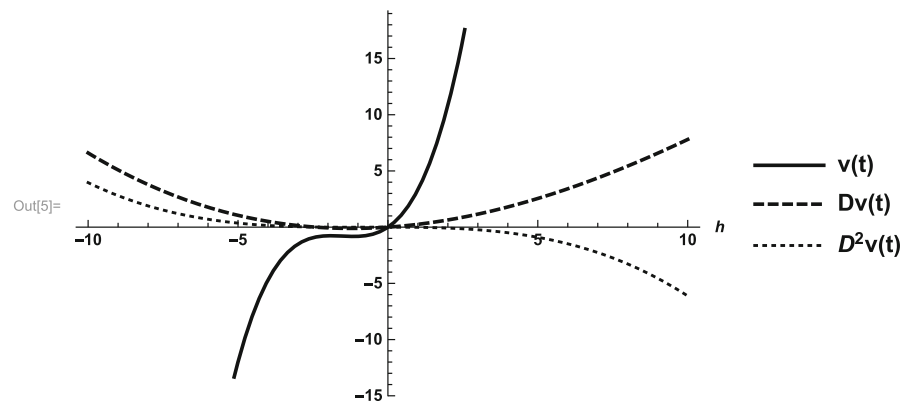

Fig. 12 The $h$-curve of the solution for the B-S equation

\subsection{Results and discussion}

The convergence region is obtained using the $h$-curve. Figure 12 shows that the convergence region of equation (4.1) is between -2 and 2. Figure 13 indicates that the FGHAM results coincide with those of HAM, HPM, MFDTM, RPS, CFADM, and the exact solution $v(x, t)$ of the $\mathrm{B}-\mathrm{S}$ equation. Figure 14 illustrates the solution $v(x, t)$ for the $\mathrm{B}-\mathrm{S}$ equation subject to the various settings of the fractional parameter $\alpha=0.25,0.5,0.75,1$, respectively. 


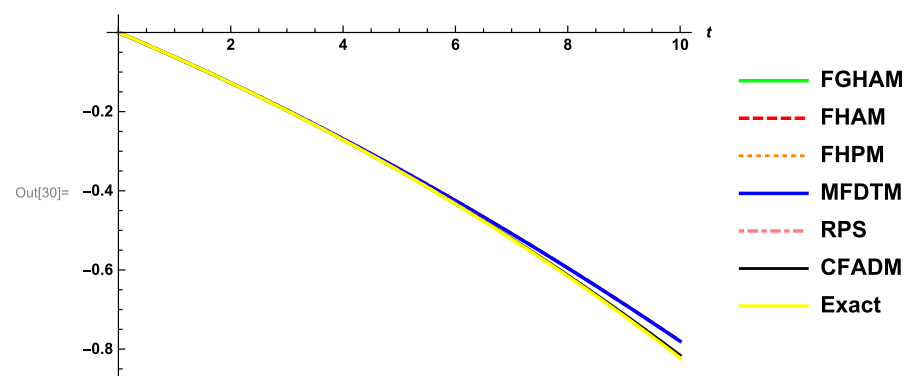

Fig. 13 Comparison among the results of FGHAM, HAM, HPM, MFDTM, RPS, CFADM, and the exact solution for the B-S equation using fractional parameter $\alpha=1$

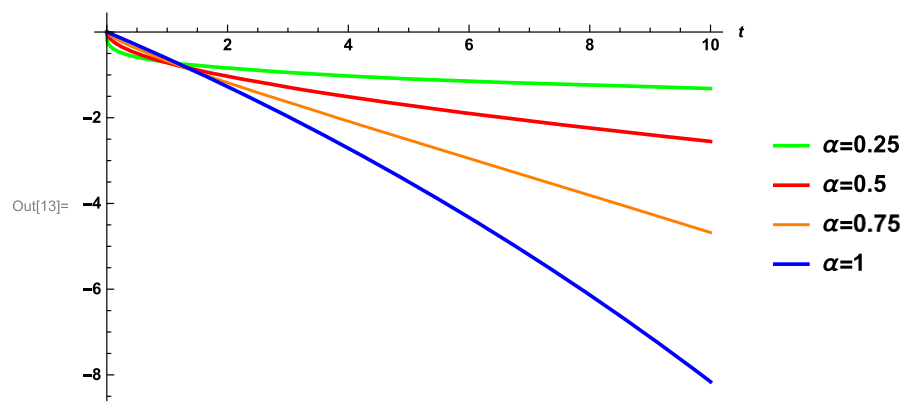

Fig. 14 Graphical illustrations of the solution for the B-S equation subject to the various settings of the fractional parameter $\alpha=0.25,0.5,0.75,1$

Figure 15 depicts the information on the financial pricing derivatives using different settings of the fractional parameter $\alpha=0.25,0.5,0.75$ and 1 , respectively.

Table 12 provides the pricing option derivatives using fractional parameter $\alpha=1$, which depicts a good agreement among the results of FGHAM, the exact solution, RPS, and CFADM, respectively.

Tables 13 and 14 provide the pricing option derivatives subject to the fractional parameter $\alpha=0.75, \alpha=0.5$, which depict a good agreement among the results of FGHAM, MFDTM, RPS, and CFADM, respectively.

\section{Fractional Black-Scholes option pricing equation}

Considering the following fractional Black-Scholes option pricing equation:

$$
\frac{\partial^{\alpha} v(x, t)}{\partial t^{\alpha}}+\frac{\sigma^{2}}{2} x^{2} \frac{\partial^{2} v(x, t)}{\partial x^{2}}+(r-\tau) x \frac{\partial v(x, t)}{\partial x}-r v(x, t)=0,0<\alpha \leq 1
$$

subject to the initial condition:

$$
v(x, 0)=\max (A x-B, 0) .
$$

Applying the fractional $G$-transform on both the sides of the equation (6.1):

$$
G_{\alpha}\left[\frac{\partial^{\alpha} v(x, t)}{\partial t^{\alpha}}+\frac{\sigma^{2}}{2} x^{2} \frac{\partial^{2} v(x, t)}{\partial x^{2}}+(r-\tau) x \frac{\partial v(x, t)}{\partial x}-r v(x, t)\right]=0
$$




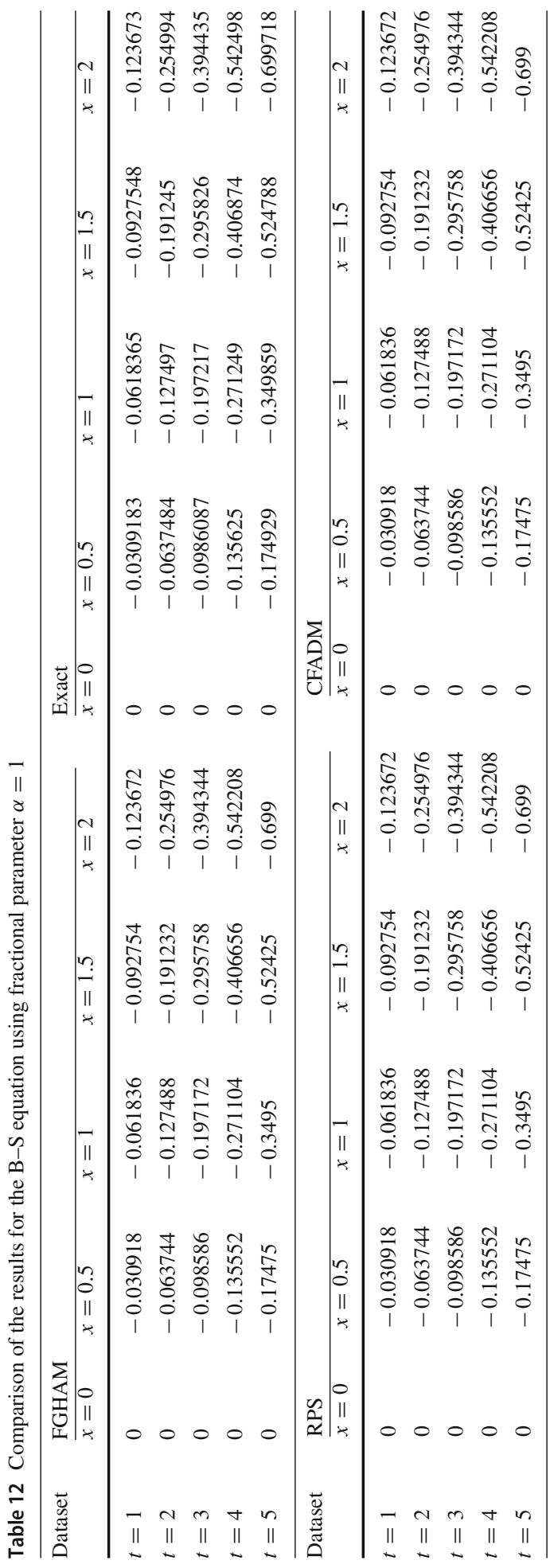




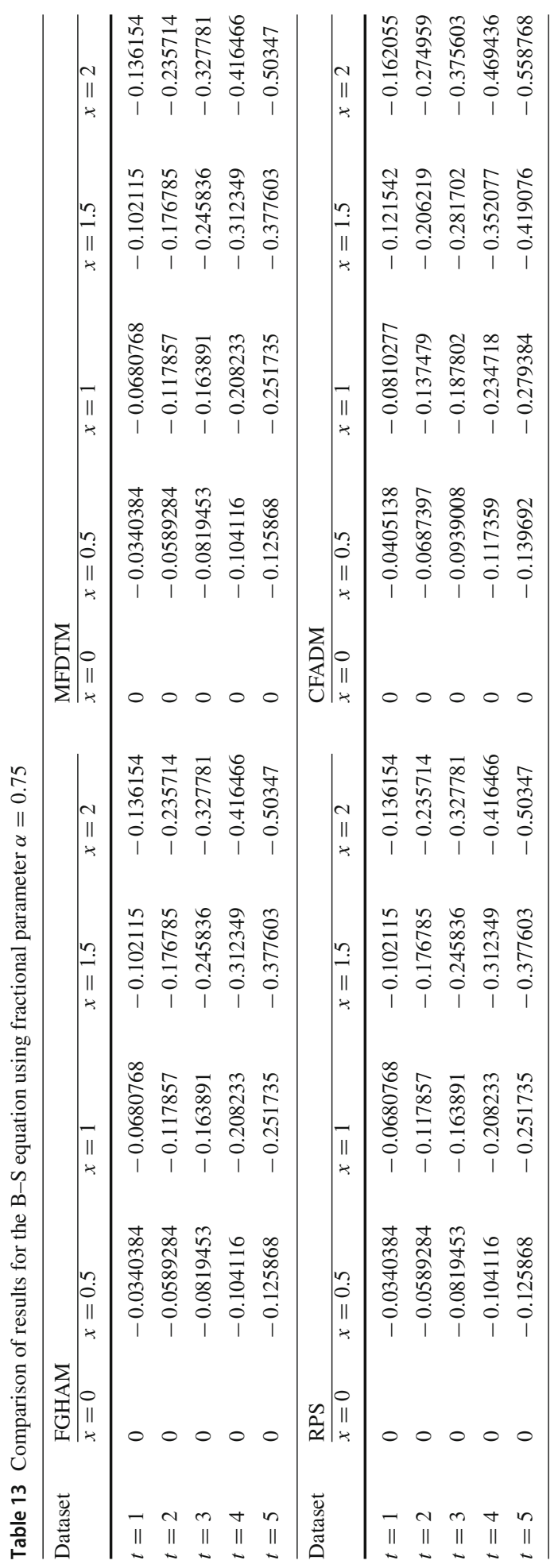




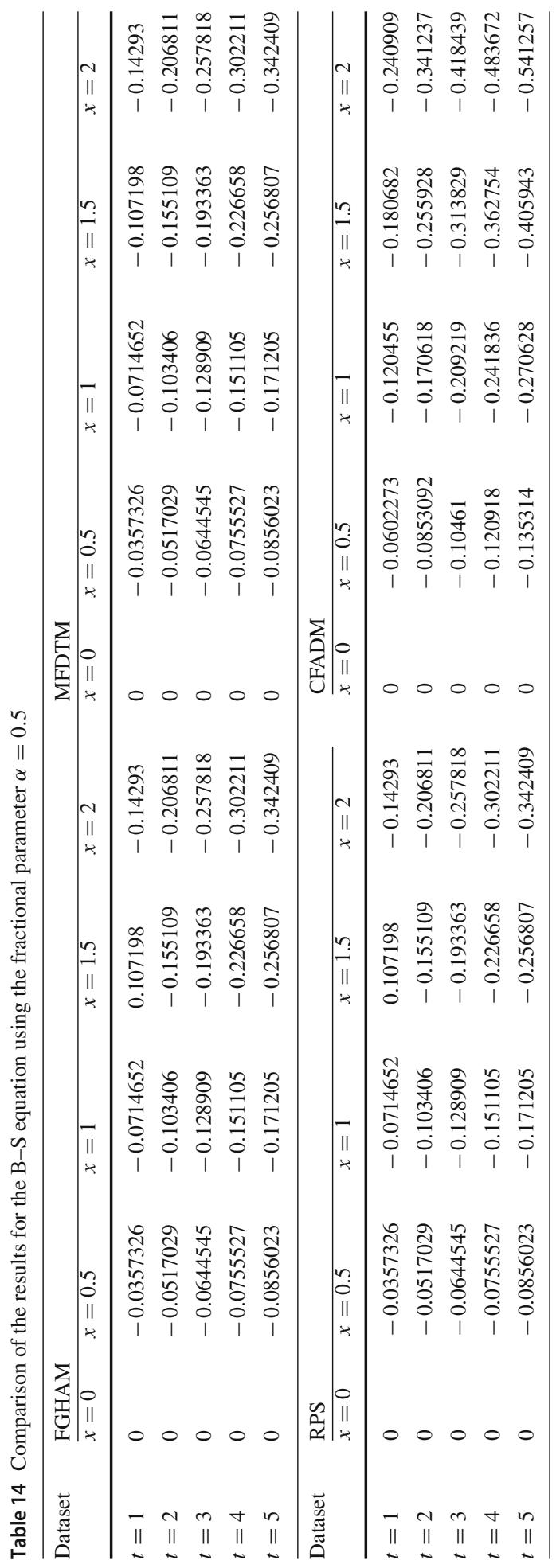




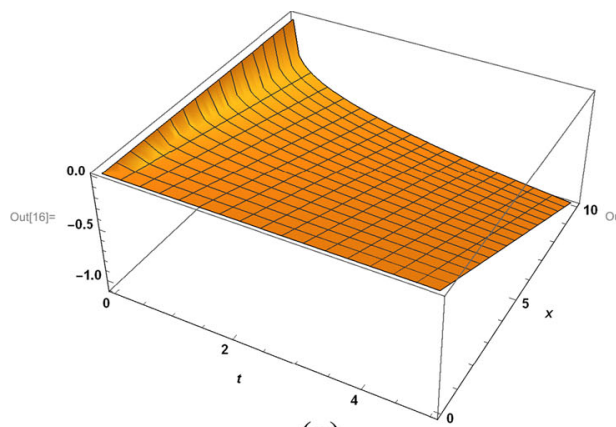

(a)

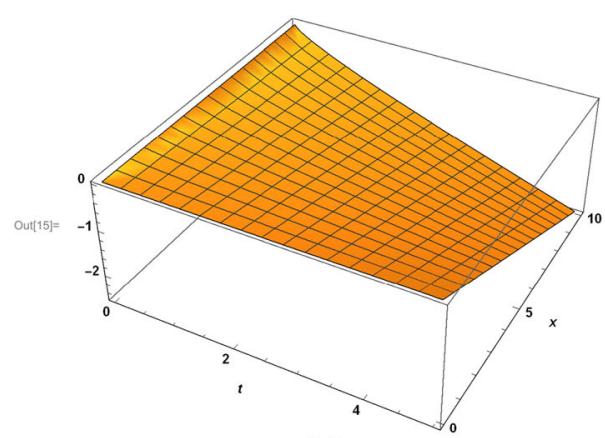

(c)

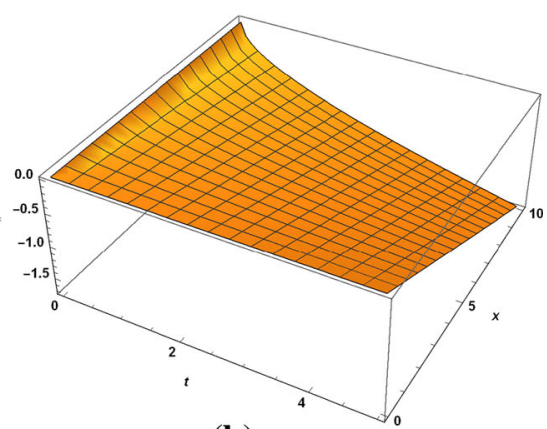

(b)

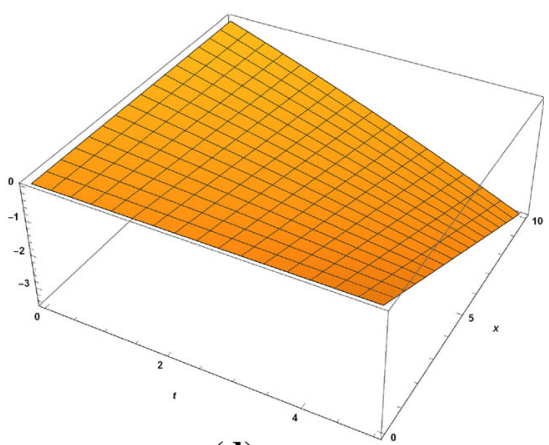

(d)

Fig. 15 The solution of $v(x, t)$ for the $\mathrm{B}-\mathrm{S}$ equation using the various settings of the fractional parameter $\alpha=0.25,0.5,0.75,1$ and the auxiliary parameter $h=-1$ are shown in $\mathbf{a}-\mathbf{d}$, respectively

$$
G_{\alpha}[v(x, t)]-u^{p+1} v(x, 0)+u^{\alpha} G_{\alpha}\left[\frac{\sigma^{2}}{2} x^{2} \frac{\partial^{2} v(x, t)}{\partial x^{2}}+(r-\tau) x \frac{\partial v(x, t)}{\partial x}-r v(x, t)\right]=0 .
$$

Applying FGHAM:

$$
v_{n}(x, t)=\chi_{n} v_{n-1}(x, t)+h G_{\alpha}^{-1}\left[R_{n}\left(\mathbf{v}_{\mathbf{n}-1}, x, t\right)\right],
$$

where

$$
\begin{aligned}
R_{n}\left[\mathbf{v}_{\mathbf{n}-1}, x, t\right]= & G_{\alpha}\left[v_{n-1}(x, t)\right]-\left(1-\chi_{n}\right) u^{p+1} v(x, 0) \\
& +u^{\alpha} G_{\alpha}\left[\frac{\sigma^{2}}{2} x^{2} \frac{\partial^{2} v(x, t)}{\partial x^{2}}+(r-\tau) x \frac{\partial v(x, t)}{\partial x}-r v(x, t)\right] .
\end{aligned}
$$

Solving the above equation for $n=1,2,3, \ldots$

$$
\begin{aligned}
& v_{0}(x, t)=\max (A x-B, 0) \\
& \left.v_{1}(x, t)=h \frac{t^{\alpha}}{\Gamma(\alpha+1)}[(r-\tau)] x \max (A, 0)-r \max (A x-B, 0)\right] \\
& v_{2}(x, t)=(h+1) v_{1}(x, t)+h^{2} \frac{t^{2 \alpha}}{\Gamma(2 \alpha+1)}\left[r^{2} \max (A x-B)-\left(r^{2}-\tau^{2}\right) x \max (A, 0)\right] \\
& v_{3}(x, t)=(h+1) v_{2}(x, t)+h^{2}(h+1) \frac{t^{2 \alpha}}{\Gamma(2 \alpha+1)}\left[r^{2} \max (A x-B, 0)-\left(r^{2}-\tau^{2}\right) x \max (A, 0)\right]
\end{aligned}
$$




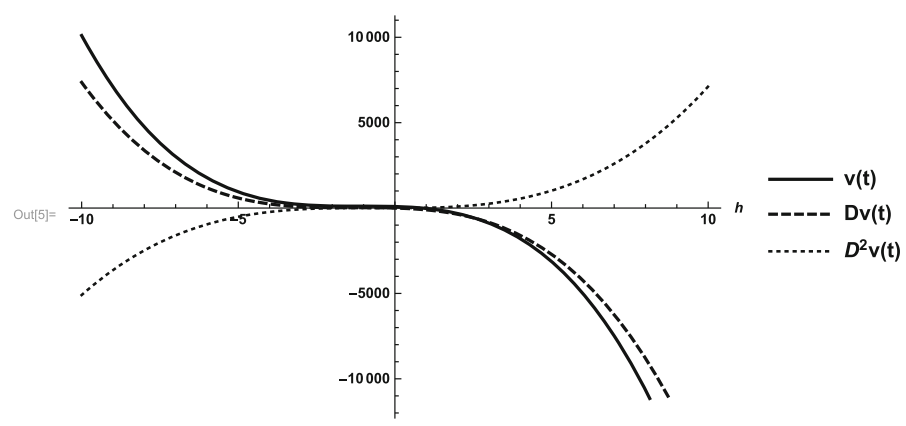

Fig. 16 The $h$-curve of the solution for the B-S equation

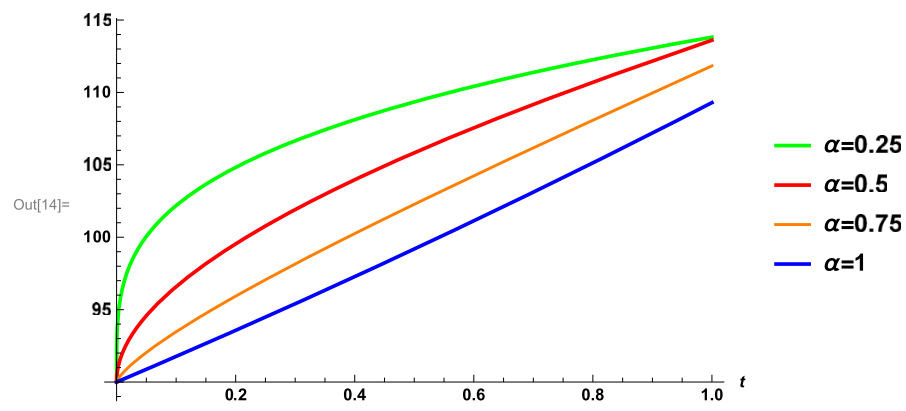

Fig. 17 A graphical illustration of the solution for the B-S equation subject to the various settings of the fractional parameter $\alpha=0.25,0.5,0.75,1$

$$
+h^{3} \frac{t^{3 \alpha}}{\Gamma(3 \alpha+1)}\left[\left(r^{3}-\tau^{3}\right) x \max (A, 0)-r^{3} \max (A x-B, 0)\right] .
$$

Similarly, $v_{4}, v_{5}, \ldots$ are estimated and the series solution is obtained as:

$$
\begin{aligned}
& v(x, t)=\sum_{n=0}^{\infty} v_{n}(x, t) \\
& v(x, t)=v_{0}(x, t)+v_{1}(x, t)+v_{2}(x, t)+v_{3}(x, t)+\cdots
\end{aligned}
$$

If $h=-1$, Eq. (6.2) can be expressed as:

$$
\begin{aligned}
v(x, t)= & \max (A x-B, 0)-\frac{t^{\alpha}}{\Gamma(\alpha+1)} \\
& +\frac{t^{2 \alpha}}{\Gamma(2 \alpha+1)}\left[r^{2} \max (A x-B)-\left(r^{2}-\tau^{2}\right) x \max (A, 0)\right] \\
& -\frac{t^{3 \alpha}}{\Gamma(3 \alpha+1)}\left[\left(r^{3}-\tau^{3}\right) x \max (A, 0)-r^{3} \max (A x-B, 0)\right]+\cdots
\end{aligned}
$$

\subsection{Results and discussion}

The convergence region is obtained using the $h$-curve. Figure 16 shows that the convergence region of equation is between -4 and 1 . Figure 17 indicates that the solution 

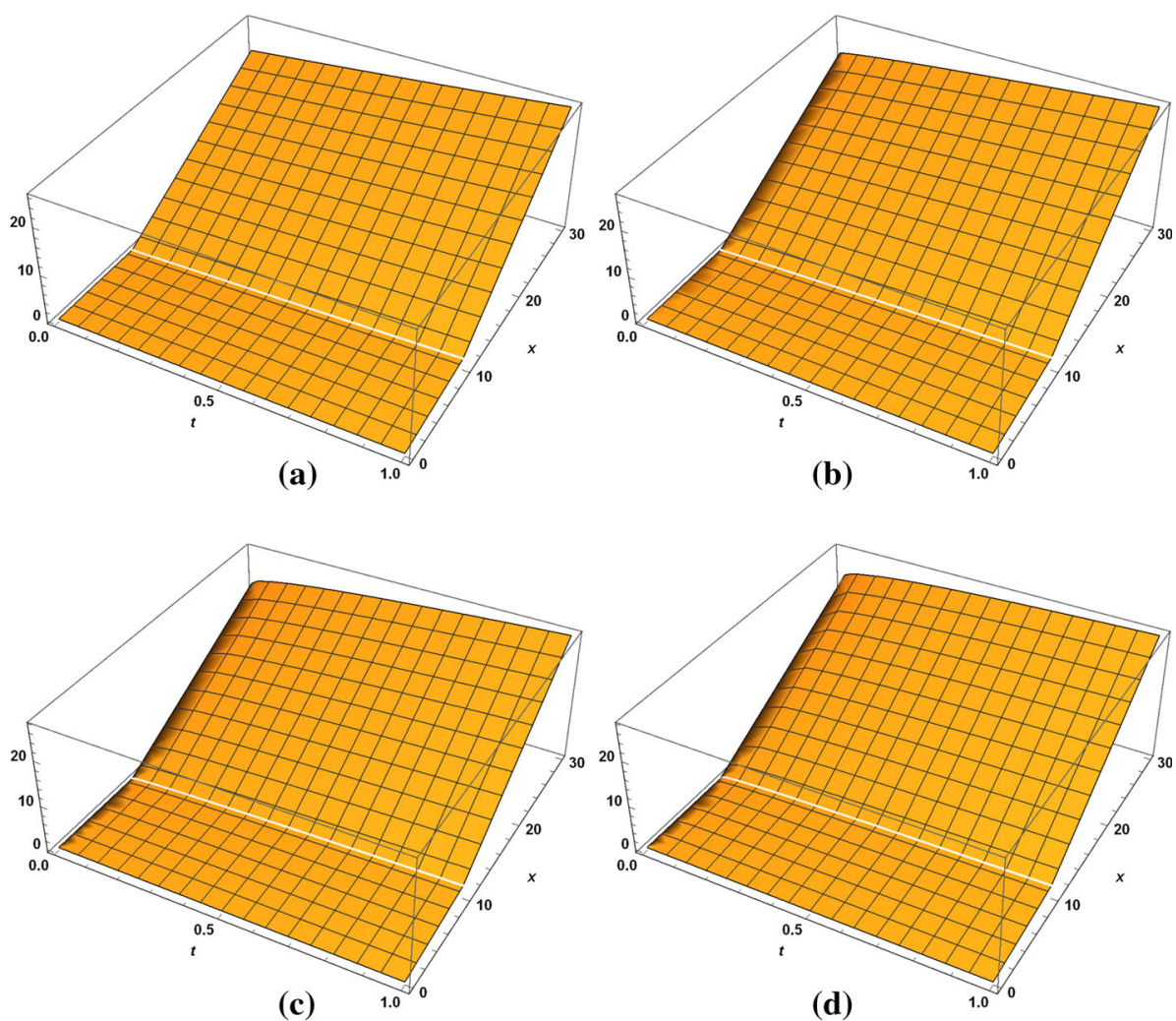

Fig. 18 The solution for the B-S equation subject to the various settings of the fractional parameter $\alpha=$ $0.25,0.5,0.75,1$ and the auxiliary parameter $h=-1$ are shown in $\mathbf{a}-\mathbf{d}$, respectively

$v(x, t)$ for the $\mathrm{B}-\mathrm{S}$ equation subject to the various settings of the fractional parameter $\alpha=0.25,0.5,0.75$ and 1 , respectively.

Figure 18 depicts the financial pricing derivatives for the different settings of the fractional parameter $\alpha=0.25,0.5,0.75$ and 1 , respectively.

\subsection{Comparison with Monte Carlo method}

To verify the accuracy of the proposed method, the numerical results of the call option obtained using FGHAM for $\alpha=1$ which is compared with the results estimated using Monte Carlo simulation (MATLAB) and the results are shown in Table 15. From Table 15, it is evident that the numerical results obtained by FGHAM show an excellent agreement with the results estimated using Monte Carlo simulation.

\section{Statistical analysis}

The statistical significance pertaining to the difference among the mean results obtained by FGHAM, exact solution, RPS, and CFADM for the B-S equation using fractional parameter 
Table 15 Comparison between FGHAM and Monte Carlo method

\begin{tabular}{lllllll}
\hline Stock Price & Strike Price & Time $(\mathrm{y})$ & Rate & Volatility & Monte Carlo & FGHAM \\
\hline 90 & 90 & 1 & 4 & 20 & 9.301401 & 9.5119 \\
95 & 95 & 1 & 4 & 20 & 10.139644 & 10.619 \\
100 & 105 & 1 & 8 & 20 & 10.348479 & 11.3 \\
105 & 105 & 1 & 6 & 25 & 12.942346 & 12.622 \\
110 & 112 & 1 & 5 & 20 & 12.101289 & 12.834 \\
115 & 115 & 1 & 10 & 30 & 17.889895 & 14.402 \\
120 & 125 & 1 & 10 & 25 & 16.888351 & 15.508 \\
125 & 125 & 1 & 8 & 20 & 16.451980 & 16.834 \\
130 & 130 & 1 & 8 & 20 & 19.299579 & 17.94 \\
135 & 140 & 1 & 8 & 20 & 20.418300 & 19.047 \\
140 & 140 & 1 & 10 & 25 & 20.596177 & 19.935 \\
145 & 150 & 1 & 1 & 20 & 11.339080 & 11.993 \\
150 & 150 & 1 & 1 & 20 & 21.837156 & 23.099 \\
155 & 160 & 1 & 4 & 20 & 23.664673 & 23.889 \\
160 & 160 & 1 & 4 & 20 & 24.550466 & 25.005 \\
\hline
\end{tabular}

Table 16 Example 1: Case 1-results of the pairwise mean difference for the B-S solutions using the $t$ test (mean difference is not statistically significant at $p<0.05$ level)

\begin{tabular}{lll}
\hline Various methods $v(x, t)$ & Mean difference & 0.95 confidence interval \\
\hline FGHAM and exact & 0.1126 & {$[-0.3417,0.5670]$} \\
FGHAM and RPS & 0 & {$[-0.4543,0.4543]$} \\
FGHAM and CFADM & 0 & {$[-0.4543,0.4543]$} \\
Exact and RPS & -0.1126 & {$[-0.5670,0.3417]$} \\
Exact and CFADM & -0.1126 & {$[-0.5670,0.3417]$} \\
RPS and CFADM & 0 & {$[-0.4543,0.4543]$} \\
\hline
\end{tabular}

$\alpha=1$ is investigated. The $t$ test (two-tailed) analysis at the 0.05 level of significance is used for the various settings of the $\mathrm{B}-\mathrm{S}$ equation discussed earlier. The results of the presented numerical examples are shown in Tables 16, 17 and 18, respectively. The comparative results of FGHAM, exact solution, RPS, and CFADM are depicted in Figures 19, 20 and 21, respectively. The null hypothesis, i.e., the mean difference of the solutions obtained for the B-S equation using FGHAM, exact solution, RPS, and CFADM, is accepted at the 0.05 level of significance (Morachan et al. 2017).

\section{Conclusions and future work}

The FGHAM approach has been successfully applied to solve the fractional non-linear B-S equation governing European option pricing. Using various plots of $h$-curves, convergence region of the solution is identified and closed form series solutions are obtained using Mittag-Leffler function, which clearly reveals the financial process. The suitable conver- 
Table 17 Example 1: Case 2-results of the pairwise mean difference for the B-S solutions using the $t$ test (mean difference is not statistically significant at $p<0.05$ level)

\begin{tabular}{lll}
\hline Various methods $v(x, t)$ & Mean difference & 0.95 confidence interval \\
\hline FGHAM and exact & 3.0843 & {$[-1.3509,7.5194]$} \\
FGHAM and RPS & 0 & {$[-4.4352,4.4352]$} \\
FGHAM and CFADM & 0 & {$[-4.4352,4.4352]$} \\
Exact and RPS & -3.0843 & {$[-7.5194,1.3509]$} \\
Exact and CFADM & -3.0843 & {$[-7.5194,1.3509]$} \\
RPS and CFADM & 0 & {$[-4.4352,4.4352]$} \\
\hline
\end{tabular}

Table 18 Example 2: results of the pairwise mean difference for B-S solutions using the $t$ test (mean difference is statistically significant at $p<0.05$ level)

\begin{tabular}{llc}
\hline Various methods $v(x, t)$ & Mean difference & 0.95 confidence interval \\
\hline FGHAM and exact & 0 & {$[-0.0243,0.0243]$} \\
FGHAM and RPS & 0 & {$[-0.0243,0.0243]$} \\
FGHAM and CFADM & 0 & {$[-0.0243,0.0243]$} \\
Exact and RPS & 0 & {$[-0.0243,0.0243]$} \\
Exact and CFADM & 0 & {$[-0.0243,0.0243]$} \\
RPS and CFADM & 0 & {$[-0.0243,0.0243]$} \\
\hline
\end{tabular}

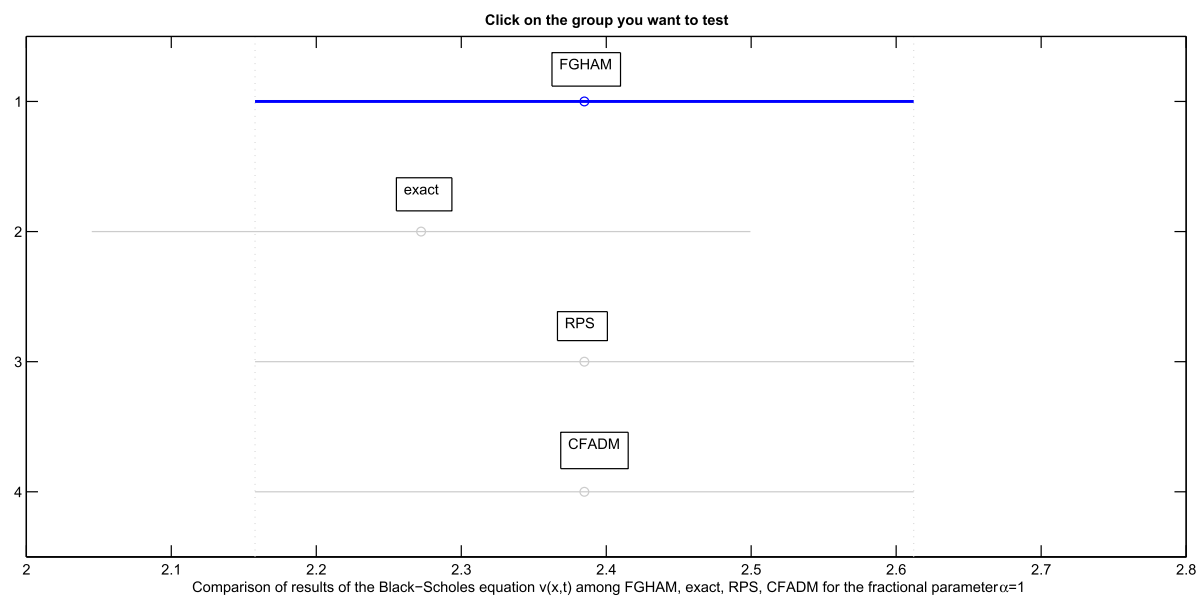

Fig. 19 Example 1: Case 1-comparison among the results of FGHAM, exact solution, RPS, and CFADM for the B-S equation

gence criterion is developed and convergence of the series solution is well established using graphical illustration. The closed form series solution of the B-S model is analyzed for various values of the fractional parameters and the results are graphically depicted. In addition, the results are validated against the various methods such as HAM, HPM, MFDTM, RPS, and CFADM, and the accuracy of the proposed FGHAM is verified. The comparative study has been carried out between FGHAM and Monte Carlo simulation and computational effi- 


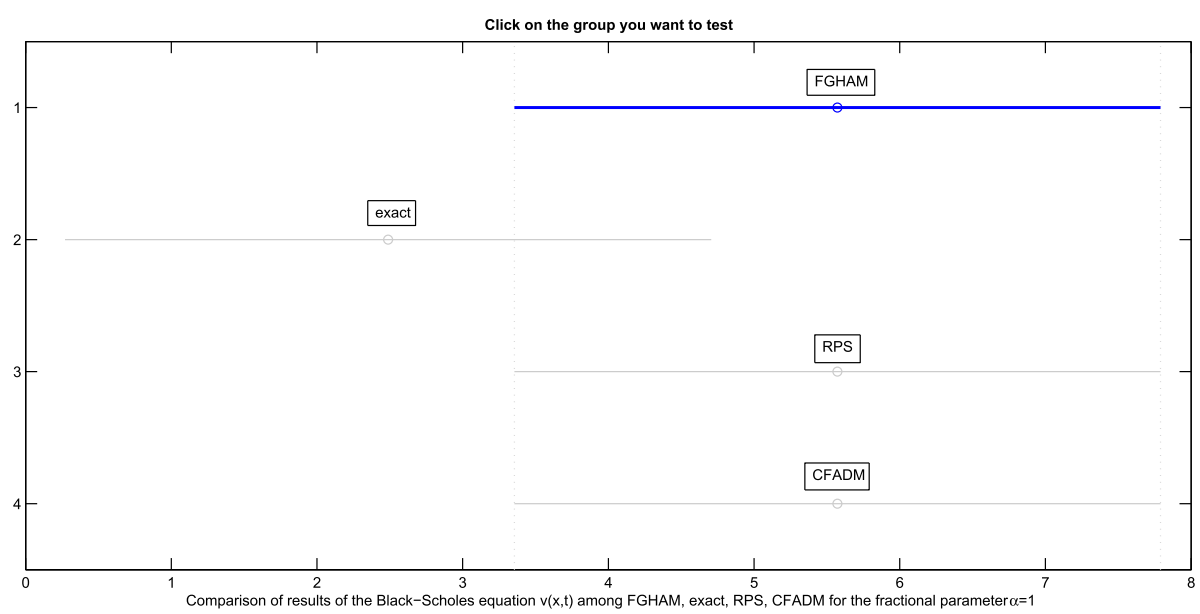

Fig. 20 Example 1: Case 2-comparison among the results of FGHAM, exact solution, RPS, and CFADM for the B-S equation

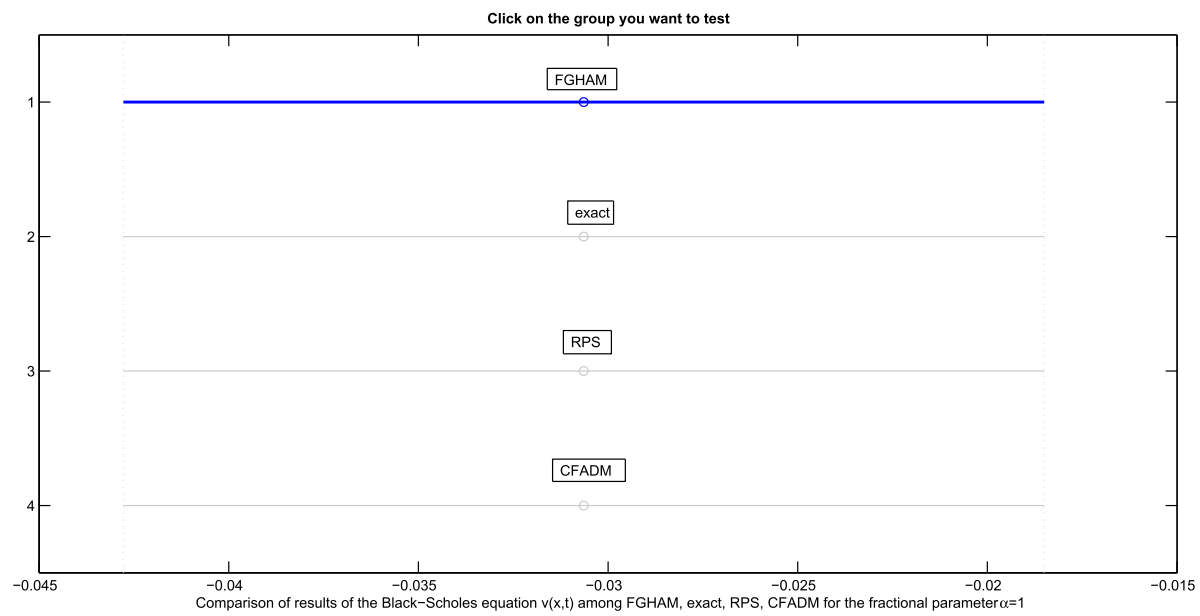

Fig. 21 Example 2: comparison among the results of FGHAM, exact solution, RPS, and CFADM for the B-S equation

ciency of the proposed method is verified. The analytical expression for the implied volatility is derived and the non-local behavior is studied for the various values of the fractional parameter. The statistical analysis has been carried out using the solutions obtained from the various methods to ascertain the effectiveness of the FGHAM approach and to tackle the B-S pricing model in financial studies. From the experimental analysis, it is evident that the proposed method accurately predicts the solution of the B-S model.

The application of FGHAM can be extended to solve the B-S model with the time variable coefficients to analyze the financial process in future. Moreover, FGHAM can be applied to solve different problems, such as the Navier-Stokes equation, epidemic models, and Pandemic model like COVID-19. The application of FGHAM can also be extended to analyze 
complicated non-linear differential equations and fractional differential equations that arise in different fields of science and engineering.

\section{References}

Bagyalakshmi M, Ganesh M, Gangadharan S (2016) Explicit solution to predict the temperature distribution and exit temperatures in a heat exchanger using differential transform method. Arab J Sci Eng 41:18251834. https://doi.org/10.1007/s13369-015-1978-1

Company R, Navarro E, Pintos JR (2008) Numerical solution of linear and nonlinear Black-Scholes option pricing equations. Comput Math Appl 56:813-821. https://doi.org/10.1016/j.camwa.2008.02.010

Dadachanji Z (2015) Derivation of the local volatility function, FX barrier options: a comprehensive guide for industry quants. Applied Quantitative finance. Palgrave Macmillan, London

Elbeleze AA, KJIJcman A, Taib B, (2013) Homotopy perturbation method for fractional Black-Scholes European option pricing equations using Sumudu transform. Math Probl Eng. https://doi.org/10.1155/2013/ 524852

Farhadi SM, Erjaee GH (2018) A new version of Black-Scholes equation presented by time-fractional derivative. Iran J Sci Technol Trans Sci 42:2159-2166. https://doi.org/10.1007/s40995-017-0244-7

Ghanbari B, Osman MS, Baleanu D (2019) Generalized exponential rational function method for extended Zakharov-Kuzetsov equation with conformable derivative. Mod Phys Lett A. https://doi.org/10.1142/ s0217732319501554

Ghandehari MAM, Ranjbar M (2014) European option pricing of fractional Black-Scholes model with new Lagrange multipliers. Comput Methods Differ Equ 2(1):1-10

Granada JRG, Sanchez CD, Sanchez HD (2017) Approximate analytical solutions for the Black-Scholes equation by homotopy perturbation method. Contemp Eng Sci 10(35):1727-1737. https://doi.org/10. 12988/ces.2017.712197

Grossinho MR, Morais E (2013) A fully nonlinear problem arising in financial modeling. Bound Value Probl 2013:146. https://doi.org/10.1186/1687-2770-2013-146

Gülkaç V (2010) The homotopy perturbation method for the Black-Scholes equation. J Stat Comput Simul 80(12):1349-1354

Haug EG, Taleb NN, and (2011) Option traders use (very) sophisticated heuristics, never the Black-ScholesMerton formula. J Econ Behav Organ 77(2):97-106

James R, Garven A (1986) Pedagogic note on the derivation of the Black-Scholes option pricing formula. Financ Rev 21(2):337-348

Javid A, Raza N, Osman MS (2019) Multi-solitons of thermophoretic motion equation depicting the wrinkle propagation in substrate-supported graphene sheets. Commun Theor Phys 71(4):362-366. https://doi. org/10.1088/0253-6102/71/4/362

Jena RM, Chakraverty S (2019) A new iterative method based solution for fractional Black-Scholes option pricing equations (BSOPE). SN Appl Sci 1:95. https://doi.org/10.1007/s42452-018-0106-8

Jumarie G (2008) Stock exchange fractional dynamics defined as fractional exponential growth driven by (usual) Gaussian white noise. Application to fractional Black-Scholes equations. Insur Math Econ 42(1):271-287

Jumarie G (2010) Derivation and solutions of some fractional Black-Scholes equations in coarse-grained space and time. Application to Merton's optimal portfolio. Comput Math Appl 59(3):1142-1164

Khan WA, Ansari FA (2016) European option pricing of fractional Black-Scholes model using Sumudu transform and its derivatives. Gen Lett Math 1(3):74-80

Kittipoom P (2018) Invariant subspace method for fractional Black-Scholes equations. Sci Asia 44:432-437. https://doi.org/10.2306/scienceasia1513-1874.2018.44.432

Kumar S, Kumar D, Singh J (2014) Numerical computation of fractional Black-Scholes equation arising in financial market. Egypt J Basic Appl Sci 1(3-4):177-183. https://doi.org/10.1016/j.ejbas.2014.10.003

Lesmana DC, Wang S (2013) An upwind finite difference method for a nonlinear Black-Scholes equation governing European option valuation under transaction costs. Appl Math Comput 219:8811-8828. https:// doi.org/10.1016/j.amc.2012.12.077

Liao SJ (1992) The proposed homotopy analysis technique for the solution of non linear problems. Ph.D. thesis, Shanghai Jiao Tong University

Liu JG, Osman MS, Zhu WH, Zhou L, Ai GP (2019) Different complex wave structures described by the Hirota equation with variable coefficients in inhomogeneous optical fibers. Appl Phys B. https://doi.org/ $10.1007 / \mathrm{s} 00340-019-7287-8$ 
Lu D, Osman MS, Khater MMA, Attia RAM, Baleanu D (2020) Analytical and numerical simulations for the kinetics of phase separation in iron $(\mathrm{Fe}-\mathrm{Cr}-\mathrm{X}(\mathrm{X}=\mathrm{Mo}, \mathrm{Cu}))$ based on ternary alloys. Phys A Stat Mech Appl 537:122634. https://doi.org/10.1016/j.physa.2019.122634

Morachan B, Gangadharan S, Ganesh M (2017) On chaotic behavior of temperature distribution in a heat exchanger. Int J Bifurc Chaos. https://doi.org/10.1142/S0218127417501681

Orlando G, Taglialatela G (2017) A review on implied volatility calculation. J Comput Appl Math 320:202-220

Osman MS (2019) New analytical study of water waves described by coupled fractional variant Boussinesq equation in fluid dynamics. Pramana J Phys 93:26. https://doi.org/10.1007/s12043-019-1785-4

Osman MS, Machado JAT (2018) The dynamical behavior of mixed-type soliton solutions described by (2 + 1)-dimensional Bogoyavlensky-Konopelchenko equation with variable coefficients. J Electromagn Waves Appl 32(11):1457-1464. https://doi.org/10.1080/09205071.2018.1445039

Osman MS, Wazwaz AM (2019) A general bilinear form to generate different wave structures of solitons for a $(3+1)$-dimensional Boiti-Leon-Manna-Pempinelli equation. Math Methods Appl Sci. https://doi.org/ 10.1002/mma.5721

Osman MS, Machado JAT, Baleanu D (2018) On nonautonomous complex wave solutions described by the coupled Schrödinger-Boussinesq equation with variable-coefficients. Opt Quantum Electron 50(73):111. https://doi.org/10.1007/s11082-018-1346-y

Osman MS, Rezazadeh H, Eslami M (2019a) Traveling wave solutions for $(3+1)$ dimensional conformable fractional Zakharov-Kuznetsov equation with power law nonlinearity. Nonlinear Eng 8(1):559-567. https://doi.org/10.1515/nleng-2018-0163

Osman MS, Lu D, Khater MMA (2019b) A study of optical wave propagation in the nonautonomous Schrödinger-Hirota equation with power-law nonlinearity. Results Phys 13:102157. https://doi.org/10. 1016/j.rinp.2019.102157

Osman MS, Lu D, Khater MMA, Attia RAM (2019c) Complex wave structures for abundant solutions related to the complex Ginzburg-Landau model. Optik 192:162927. https://doi.org/10.1016/j.ijleo.2019.06.027

Ouafoudi M, Gao F (2018) Exact solution of fractional Black-Scholes European option pricing equations. Appl Math 9:86-100. https://doi.org/10.4236/am.2018.91006

Prathumwan D, Trachoo K (2019) Application of the Laplace homotopy perturbation method to the BlackScholes model based on a European put option with two assets. Mathematics 7:310. https://doi.org/10. 3390/math7040310

Ravi Kanth ASV, Aruna K (2016) Solution of time fractional Black-Scholes European option pricing equation arising in financial market. Nonlinear Eng 5(4):269-276. https://doi.org/10.1515/nleng-2016-0052

Rezazadeh H, Osman MS, Eslami M, Mirzazadeh M, Zhou Q, Badri SA, Korkmaz A (2019) Hyperbolic rational solutions to a variety of conformable fractional Boussinesq-Like equations. Nonlinear Eng 8(1):224-230. https://doi.org/10.1515/nleng-2018-0033

Rouah FD (2014) Four derivations of the Black Scholes PDE, Mathematical finance notes. http://www.frouah. com/pages/finmath.html. Accessed July 2020

Samko SG, Kilbas AA, Marichev OI (1993) Fractional integrals and derivatives. Theory and applications. Gordon and Breach Science Publishers, Cham

Saratha SR, M B, G SSK (2020) Fractional generalised homotopy analysis method for solving nonlinear differential equations. Comput Appl Math 39:112. https://doi.org/10.1007/s40314-020-1133-9

Sawangtong P, Trachoo K, Sawangtong W, Wiwattanapataphee B (2018) The analytical solution for the BlackScholes equation with two assets in the Liouville-Caputo fractional derivative sense. Mathematics 6:129. https://doi.org/10.3390/math6080129

Song L, Wang W (2013) Solution of the fractional Black-Scholes a pedagogic note on the derivation of the Black-Scholes option pricing formula. Option pricing model by finite difference method. Abstr Appl Anal. https://doi.org/10.1155/2013/194286

Sumiati I (2019) Black-Scholes equation solution using Laplace-Adomian decomposition method. IAENG Int J Comput Sci 46:4

Uddin M, Taufiq M (2019) Approximation of time fractional Black-Scholes equation via radial kernels and transformations. Fract Differ Calc 9(1):75-90. https://doi.org/10.7153/Fdc-2019-09-06

Wyss W (2000) The fractional Black-Scholes equation. Fract Calc Appl Anal Theory Appl 3(1):51-61

Xiaozhong Y, Lifei W, Shuzhen S, Xue Z (2016) A universal difference method for time-space fractional Black-Scholes equation. Adv Differ Equ 2016:71. https://doi.org/10.1186/s13662-016-0792-8

Yavuz M, Ozdemir N (2018) A different approach to the European option pricing model with new fractional operator. Math Model Nat Phenom 13:12. https://doi.org/10.1051/mmnp/2018009

Publisher's Note Springer Nature remains neutral with regard to jurisdictional claims in published maps and institutional affiliations. 


\section{Affiliations}

S. R. Saratha ${ }^{1}$ - G. Sai Sundara Krishnan ${ }^{2}$ - M. Bagyalakshmi ${ }^{3}$. Chee Peng Lim ${ }^{4}$

M. Bagyalakshmi

bagyalakshmisankar@gmail.com

S. R. Saratha

ammusaratha@gmail.com

G. Sai Sundara Krishnan

ssk.amcs@psgtech.ac.in

Chee Peng Lim

chee.lim@deakin.edu.au

1 Department of Mathematics, Kumaraguru College of Technology, Coimbatore, Tamilnadu, India

2 Department of Applied Mathematics and Computational Sciences, PSG College of Technology, Coimbatore, Tamilnadu, India

3 Department of Mathematics, PSG College of Technology, Coimbatore, Tamilnadu, India

4 Institute for Intelligent Systems Research and Innovation, Deakin University, Geelong, Victoria, Australia 